\title{
Regularity of solutions of the fractional porous medium flow
}

Received Aprit 16, 2012 and in revised form Jamuary 5, 2013

Abstract. We study a porous medium equation with nonlocal diffusion effects given by an inverse fractional Laplacian operator. The precise model is

$$
u_{t}=\nabla \cdot\left(u \nabla(-\Delta)^{-s} u\right), \quad 0<s<1 .
$$

The problem is posed in $\left\{x \in \mathbb{R}^{N}, t \in \mathbb{R}\right\}$ with nonnegative initial data $u(x, 0)$ that are integrable and decay at infinity. A previous paper has established the existence of mass-preserving, nonnegative weak solutions satisfying energy estimates and finite propagation. As main results we establish the boundedness and $C^{\alpha}$ regularity of such weak solutions. Finally, we extend the existence theory to all nonnegative and integrable initial data.

Keywords. Porous medium equation, fractional Laplacian, nonlocal operator, regularity

\section{Introduction}

This paper is devoted to the study of the regularity properties of weak solutions of a model of porous medium equation that includes nonlocal effects through an integral relation of pressure to density. This allows one to account for long-range effects.

Let us recall the typical derivation of the porous medium equation (cf. [1, 20]). We consider a gas propagating in a homogeneous porous medium; its dynamics is described by first assuming conservation of mass

$$
\partial_{t} u+\nabla \cdot(\mathbf{v} u)=0
$$

where $u(x, t) \geq 0$ denotes the density of the gas and $\mathbf{v}(x, t)$ is the (locally averaged) velocity. We then postulate that the motion proceeds according to Darcy's law so that $\mathbf{v}=-\nabla p$, where the velocity potential is interpreted as pressure. Finally, some barotropic

L. A. Caffarelli: School of Mathematics, University of Texas at Austin, 1 University Station, C1200, Austin, TX 78712-1082, USA, and Institute for Computational Engineering and Sciences; e-mail: caffarel@math.utexas.edu

F. Soria and J. L. Vázquez: Departamento de Matemáticas, Universidad Autónoma de Madrid, 28049 Madrid, Spain, and ICMAT; e-mail: fernando.soria@uam.es, juanluis.vazquez@uam.es

Mathematics Subject Classification (2010): 35K55, 35K65, 76S05 
state law for gases implies that $p$ is a monotone function of $u, p=f(u)$. In this way we get the equation

$$
\partial_{t} u=\nabla \cdot(u \nabla f(u)) .
$$

The simplest case (called isothermal) is $p=u$ and in that case we arrive at the equation $\partial_{t} u=c \Delta u^{2}$, which appears in a different context as a model in groundwater infiltration, and is called Boussinesq's equation $[2,4]$.

The novelty of our present model consists in relating $p$ to $u$ through a linear integral operator that makes a kind of average of the space distribution $u(\cdot, t)$,

$$
p(x, t)=\mathcal{L} u(x, t), \quad \mathcal{L} u(x, t):=\int L(x-y) u(y, t) d y .
$$

In particular, the positive kernel $L$ is locally integrable and decays slowly at infinity to represent "long-range" interactions. To be specific, we will work in $\mathbb{R}^{N}$, we will take $L(x)=c|x|^{-N+2 s}$, which is equivalent to saying that $p$ is given as an inverse fractional Laplacian, i.e., $p=(-\Delta)^{-s} u$, and we consider $0<s<1$.

In a previous paper [11] we have introduced this model and proved existence of weak solutions $u \geq 0$ for the Cauchy problem

$$
u_{t}=\nabla \cdot(u \nabla p), \quad p=\mathcal{L} u=(-\Delta)^{-s} u, 0<s<1,
$$

posed for $x \in \mathbb{R}^{N}, N \geq 1$, and $t>0$, with initial conditions

$$
u(x, 0)=u_{0}(x), \quad x \in \mathbb{R}^{N},
$$

where $u_{0}$ is a nonnegative and integrable function in $\mathbb{R}^{N}$ decaying as $|x| \rightarrow \infty$.

Let us point out that equations of the more general form $u_{t}=\nabla \cdot(\sigma(u) \nabla \mathcal{L} u)$ have appeared recently in a number of applications in particle physics. Thus, Giacomin and Lebowitz consider in [13] a lattice gas with general short-range interactions and a Kac potential $J_{\gamma}(r)$ of range $\gamma^{-1}, \gamma>0$. Scaling spacelike with $\gamma^{-1}$ and timelike with $\gamma^{-2}$, and passing to the limit $\gamma \rightarrow 0$, the macroscopic density profile $\rho(r, t)$ satisfies the equation

$$
\frac{\partial \rho}{\partial t}=\nabla \cdot\left[\sigma_{s}(\rho) \nabla \frac{\delta F(\rho)}{\delta \rho}\right] .
$$

Here $J$ is a kernel, $F(\rho)=\int f_{s}(\rho(r)) d r-\frac{1}{2} \iint J\left(r-r^{\prime}\right) \rho(r) \rho\left(r^{\prime}\right) d r d r^{\prime}$, where $f_{s}(\rho)$ is the (strictly convex) free energy density of the reference system, and $\sigma_{s}(\rho)$ is the mobility of the system with only short-range interactions. See also [14] and the review paper [16]. The model is used to study phase segregation in [15].

Further motivations for model (1.3) can be found in [11] and [22], which contain references to applications in dislocation dynamics and in superconductivity, as well as current mathematical progress.

Mathematical results. Paper [11] contains the proof of existence of a weak solution of Problem (1.2)-(1.3) when $u_{0}$ is a bounded function and has exponential decay at infinity. Moreover, a number of basic properties are proved, like energy estimates, bounds in the 
$L^{p}$ spaces, and the property of finite propagation that says that compactly supported data produce solutions whose support is compact in space for every positive time. However, the question of uniqueness of weak solutions is a pending open problem (in more than one space dimension). Comparison theorems, a crucial tool in parabolic equations, are only available under special circumstances (i.e., for so-called true super- or subsolutions). The asymptotic behaviour of the solutions as $t \rightarrow \infty$ has been studied by two of the present authors in [12] using obstacle problems and entropy estimates.

The regularity theory that we develop in the present paper is as follows:

- If $u$ has initial data in $L^{1} \log L$, then it becomes instantaneously bounded.

- If $u$ has initial data in $L^{1}$, then it falls into the previous case.

- Bounded solutions are continuous with a modulus of continuity.

After some preliminaries in Section 2, and the needed theory of bilinear forms in Section 3, the following boundedness result is proved in Section 4:

Theorem 1.1. Let $u$ be a weak energy solution of Problem (1.2)-(1.3) with $u_{0} \in$ $L^{1}\left(\mathbb{R}^{N}\right) \cap L^{\infty}\left(\mathbb{R}^{N}\right)$. Then there exists a positive constant $C$ such that for every $t>0$,

$$
\sup _{x \in \mathbb{R}^{N}}|u(x, t)| \leq C t^{-\alpha}\left\|u_{0}\right\|_{L^{1}\left(\mathbb{R}^{N}\right)}^{\gamma}
$$

with precise exponents $\alpha=N /(N+2-2 s), \gamma=(2-2 s) /(N+2-2 s)$. The constant $C$ depends only on $N \geq 1$ and $s \in(0,1)$.

The class of weak energy solutions that we use is described in Section 2. Note that this estimate and conservation of mass imply a decay for all intermediate norms $L^{p}$ with $1<p<\infty$ :

$$
\|u(\cdot, t)\|_{p} \leq C_{p} t^{-\alpha_{p}}\left\|u_{0}\right\|_{L^{1}\left(\mathbb{R}^{N}\right)}^{\gamma_{p}},
$$

where $\alpha_{p}=\alpha(p-1) / p$ and $\gamma_{p}=(1+\gamma(p-1)) / p$

The main result of the present paper is the $C^{\alpha}$ regularity of the solutions:

Theorem 1.2. Let $u \geq 0$ be a bounded weak energy solution defined in a space-time strip $S=\mathbb{R}^{N} \times\left[T_{1}, T_{2}\right] \subset \mathbb{R}^{N+1}$. Let $s \in(0,1), s \neq 1 / 2$. Then $u$ is $C^{\alpha}$ continuous in any interior subdomain $S^{\prime} \Subset S$ for some exponent $\alpha(N, s) \in(0,1)$ and a constant that depends also on the distance from $S^{\prime}$ to $S$ and the bounds on $u$ in $L^{\infty}(S)$ and $L^{\infty}\left(T_{1}, T_{2} ; L^{1}\left(\mathbb{R}^{N}\right)\right)$.

The proof of this result takes up Sections 5 to 12 . In the range $0<s<1 / 2$ it uses a number of techniques that are becoming classical in the study of regularity of nonlocal diffusion problems, but it is complicated by having to take into account both the nonlinearity and the possible degeneracy. Section 12 covers the more difficult range $s>1 / 2$. The regularity result in that case uses transport ideas in the form of a geometrical transformation to absorb the uncontrolled growth of one of the integrals that appear in the iterated energy estimates. The case $s=1 / 2$ has new difficulties and is not covered in this paper. We conjecture that the $C^{\alpha}$ regularity is then also true. 
As a consequence of these results, in Section 13 we complete the existence theory by constructing a continuous weak solution for any initial data $u_{0} \in L^{1}\left(\mathbb{R}^{N}\right), u_{0} \geq 0$ (see a detailed statement in Theorem 13.1).

Notations. We will refer to (1.2) as the FPME (with F for fractional). We will use the notation $(-\Delta)^{s}$ with $0<s<1$ for the fractional powers of the Laplace operator defined on the Schwartz class of functions in $\mathbb{R}^{N}$ by Fourier transform and extended in a natural way to functions in the Sobolev space $H^{2 s}\left(\mathbb{R}^{N}\right)$. Technical reasons imply that in one space dimension the restriction $s<1 / 2$ will be observed. The inverse operator is denoted by $\mathcal{L}_{s}=(-\Delta)^{-s}$ and can be realized by convolution

$$
\mathcal{L}_{s} u=L_{s} * u, \quad L_{s}(x)=c(N, s)|x|^{-N+2 s} .
$$

$\mathcal{L}_{s}$ is a positive self-adjoint operator. We will write $\mathcal{H}_{s}=\mathcal{L}_{s}^{1 / 2}$, which has kernel $L_{s / 2}$. The subscript $s$ will be omitted when $s$ is fixed and known. For functions that depend on $x$ and $t$, convolution is applied for every fixed $t$ with respect to the space variables. We then use the abbreviated notation $u(t)=u(\cdot, t)$.

For a measurable $u \geq 0$ and for $k>0$ we write $u_{k}^{+}=(u-k)^{+}=\max \{u-k, 0\}$, and $u_{k}^{-}=\min \{u-k, 0\}$ so that $u_{k}^{+} \geq 0 \geq u_{k}^{-}$, the supports of $u_{k}^{+}$and $u_{k}^{-}$intersect only at points where $u=0$, and also $u=k+u_{k}^{+}+u_{k}^{-}$. We will use similar notations: $u_{\varphi}^{+}=(u-\varphi)^{+}, u_{\varphi}^{-}=(u-\varphi)^{-}$when $\varphi$ is a function and not just a constant, and then we may split $u$ as follows: $u=\varphi+u_{\varphi}^{+}+u_{\varphi}^{-}$.

\section{Preliminaries. Existence and basic estimates}

Definition. We say that $u$ is a weak solution of Problem (1.2)-(1.3) in $Q_{T}=\mathbb{R}^{N} \times(0, T)$ with initial data $u_{0} \in L^{1}\left(\mathbb{R}^{N}\right)$ if $u \in L^{1}\left(Q_{T}\right), \mathcal{L}(u) \in L_{\text {loc }}^{1}\left(0, T ; W_{\text {loc }}^{1,1}\left(\mathbb{R}^{N}\right)\right)$, and $u \nabla \mathcal{L}(u) \in L^{1}\left(Q_{T}\right)$, and the identity

$$
\iint u\left(\eta_{t}-\nabla \mathcal{L}(u) \cdot \nabla \eta\right) d x d t+\int u_{0}(x) \eta(x, 0) d x=0
$$

holds for all continuously differentiable test functions $\eta$ in $Q_{T}$ that are compactly supported in the space variable and vanish near $t=T$.

We will write $Q$ instead of $Q_{T}$ when $T=\infty$. The following results have been proved in [11].

Theorem 2.1. Let $u_{0} \in L^{\infty}\left(\mathbb{R}^{N}\right), u_{0} \geq 0$, be such that

$$
u_{0}(x) \leq A e^{-a|x|} \quad \text { for some } A, a>0 .
$$

Then there exists a weak solution $u$ of (1.2) with initial data $u_{0}$. Moreover, $u \in$ $L^{\infty}\left(0, \infty ; L^{1}\left(\mathbb{R}^{N}\right)\right), u \in L^{\infty}(Q), \nabla \mathcal{H}(u) \in L^{2}(Q)$. Moreover, for all $t>0$ we have conservation of mass:

$$
\int_{\mathbb{R}^{N}} u(x, t) d x=\int_{\mathbb{R}^{N}} u_{0}(x) d x
$$


as well as the $L^{\infty}$ bound: $\|u(t)\|_{\infty} \leq\left\|u_{0}\right\|_{\infty}$. The solution decays exponentially as $|x| \rightarrow \infty$. The first energy inequality holds in the form

$$
\int_{0}^{t} \int_{\mathbb{R}^{N}}|\nabla \mathcal{H} u|^{2} d x d s+\int_{\mathbb{R}^{N}} u(t) \log (u(t)) d x \leq \int_{\mathbb{R}^{N}} u_{0} \log \left(u_{0}\right) d x,
$$

while the second says that for all $0<t_{1}<t_{2}<\infty$,

$$
\int_{t_{1}}^{t_{2}} \int_{\mathbb{R}^{N}} u|\nabla \mathcal{L} u|^{2} d x d t+\frac{1}{2} \int_{\mathbb{R}^{N}}\left|\mathcal{H} u\left(t_{2}\right)\right|^{2} d x \leq \frac{1}{2} \int_{\mathbb{R}^{N}}\left|\mathcal{H} u\left(t_{1}\right)\right|^{2} d x .
$$

Other properties of the constructed solutions. Here are some of the most useful:

- Translation invariance: The equation is invariant under translations in space and time, and this property reflects in the set of weak solutions.

- Scaling: Moreover, the equation is invariant under a subgroup of the group of dilations in $(u, x, t)$, and this implies a scaling property for the set of solutions. Namely, if $u(x, t)$ is a weak solution as described in the existence theorem, with initial data $u_{0}(x)$, and $A, B, C$ are positive constants, then $\widehat{u}(x, t)=A u(B x, C t)$ is again a weak solution on the condition that $A=C B^{-2+2 s}$. It has initial data $\widehat{u}_{0}(x)=A u_{0}(B x)$.

- Conservation of sign: $u_{0} \geq 0$ implies that $u(t) \geq 0$ for all times.

- $L^{p}$ estimates: The $L^{p}$ norm of the solutions, $1<p \leq \infty$, does not increase in time.

- Finite propagation: Compactly supported initial data $u_{0}(x)$ give rise to solutions $u(x, t)$ that have the same property for all positive times, i.e., the support of $u(\cdot, t)$ is contained in a finite ball $B_{R(t)}(0)$ for any $t>0$.

- A standard comparison result for parabolic equations does not work in general. This is one of the main technical difficulties in the study of this equation. In fact, special situations are found in [11] where some comparison holds by using so-called true superand subsolutions.

Weak energy solutions. The constructed solutions are limits of smooth functions for which the energy inequalities are justified. We will need this fact and also similar integrations by parts involved in the new energy inequalities. In particular, we want the weak solution to satisfy the identities

$$
\iint u\left(\eta_{t}-\mathcal{B}_{r}(u, \eta)\right) d x d t+\int u_{0}(x) \eta(x, 0) d x=0,
$$

where $\mathcal{B}_{r}$ is the bilinear form that will be defined in the next section, $r=1-s$ and $\eta \in L^{2}\left(0, T ; H^{r}\left(\mathbb{R}^{N}\right)\right), \eta$ bounded, $\eta_{t} \in L^{2}\left(Q_{T}\right)$. This class of solutions can be called weak energy solutions. Below (see (4.2), (4.4)), we will need a version of this definition which consists in using $\eta=f(u)$ and integrating in time to get

$$
\left.\int F(u(t)) d x\right|_{t_{1}} ^{t_{2}}+\int_{t_{1}}^{t_{2}} \int \nabla[f(u)] u \nabla \mathcal{L} u d x d t=0
$$

with $f$ smooth and bounded, $F(s)=\int^{s} f(s) d s$, and $0 \leq t_{1}<t_{2} \leq T$. Note that we do not need to assume regularity for $\eta_{t}$. The constructed bounded solutions are energy solutions in this sense. We will also use $\eta=f(u / \varphi)$, where $f$ is as before and $\varphi(x)$ is a smooth positive function that does not vanish (see (6.2) and later). 


\section{Bilinear forms}

Before proceeding with the study of the boundedness and regularity properties, we need some results on fractional operators. The bilinear form associated to the space $H^{r}\left(\mathbb{R}^{N}\right)=$ $W^{r, 2}\left(\mathbb{R}^{N}\right), 0<r<1$, is

$$
\mathcal{B}_{r}(v, w)=C_{N, r} \iint(v(x)-v(y)) \frac{1}{|x-y|^{N+2 r}}(w(x)-w(y)) d x d y
$$

where $C_{N, r}$ denotes a normalizing constant. Using smooth and compactly supported functions, one can easily obtain the identity

$$
\mathcal{B}_{r}(v, w)=\int \hat{v}(\xi) \overline{\hat{w}(\xi)}|\xi|^{2 r} d \xi
$$

so that $\|v\|=\|v\|_{L^{2}}+\mathcal{B}_{r}(v, v)$ defines a norm in $H^{r}\left(\mathbb{R}^{N}\right)$. We will omit the subscript and write $\mathcal{B}$ instead of $\mathcal{B}_{r}$ when the context is clear.

Corollary 3.1. (a) If $v$ is a monotone function of $w$, i.e., if $v=G(w)$ with $G^{\prime} \geq 0$, then $\mathcal{B}(v, w) \geq 0$.

(b) If $G^{\prime}(s) \leq C$ for some constant $C>0$, then $\mathcal{B}(G(w), w) \leq C \mathcal{B}(w, w)$.

Proposition 3.2. For every $u, v \in H^{1}\left(\mathbb{R}^{N}\right)=W^{1,2}\left(\mathbb{R}^{N}\right)$ we have

$$
\mathcal{B}_{r}(v, w)=C \iint \nabla v(x) \frac{1}{|x-y|^{N-2+2 r}} \nabla w(y) d x d y .
$$

Proof. Prove it first for $C_{c}^{\infty}$ functions and use a smoothing and truncation of the kernel. Then pass to the limit.

Remark. Since the weak formulation of the FPME leads to an expression of this latter form with kernel $L=c|x-y|^{-N+2 s}$, we will put below $r=1-s$. Actually, all that we will use in Sections 4 and later, in accordance with Proposition 3.2, is two kernels $L$ and $K$ such that $L, K \geq 0$ and $\Delta L=K$, as well as the bilinear forms associated to the pairings

$$
\nabla(\cdot) \cdot L \cdot \nabla(\cdot) \leftrightarrow \text { difference } \cdot K \cdot \text { difference, }
$$

which is a short way of writing the equivalence of formulas (3.1), (3.2). In later calculations we will also use the following positivity properties of the integration of these kernels applied to truncations of functions.

Lemma 3.1. Let $u \in H^{r}\left(\mathbb{R}^{N}\right)$ and $u_{k}^{+} \in H^{r}\left(\mathbb{R}^{N}\right)$ for some $k>0$. Then

$$
\mathcal{B}_{r}\left(u_{k}^{+}, u\right) \geq \mathcal{B}_{r}\left(u_{k}^{+}, u_{k}^{+}\right)
$$


Proof. If $K_{r}(x, y)=|x-y|^{-(N+2 r)}$ is the kernel of $\mathcal{B}_{r}$, we have

$$
\begin{aligned}
\mathcal{B}_{r}\left(u_{k}^{+}, u\right)-\mathcal{B}_{r}\left(u_{k}^{+}, u_{k}^{+}\right) & =\mathcal{B}_{r}\left(u_{k}^{+}, u-u_{k}^{+}\right)=\mathcal{B}_{r}\left(u_{k}^{+}, k+u_{k}^{-}\right) \\
& =\iint\left(u_{k}^{+}(x)-u_{k}^{+}(y)\right) K_{r}(x, y)\left(u_{k}^{-}(x)-u_{k}^{-}(y)\right) d x d y .
\end{aligned}
$$

Now, given the fact that $u^{+}(x) u^{-}(x)=0$ a.e. and symmetry in $x, y$, the last integral equals

$$
-2 \int u_{k}^{+}(x) u_{k}^{-}(y) K(x, y) d x d y \geq 0 .
$$

We will also need the following embedding inequality.

Lemma 3.2. For every $u \in L^{1}\left(\mathbb{R}^{N}\right) \cap H^{r}\left(\mathbb{R}^{N}\right), u \geq 0$, we have

$$
\int u^{q} d x \leq C\|u\|_{L^{1}}^{\theta}\|u\|_{H^{r}}^{2}
$$

where $\theta=2 r / N, q=2+\theta=2+2 r / N$, and $C=C(N, r)>0$. Moreover, for every $u \in L^{2}\left(\mathbb{R}^{N}\right) \cap H^{r}\left(\mathbb{R}^{N}\right), u \geq 0$, we have

$$
\int u^{q_{1}} d x \leq C\|u\|_{L^{2}}^{\theta}\|u\|_{H^{r}}^{2},
$$

where $\theta=4 r / N, q_{1}=2+4 r / N$, and $C=C(N, r)>0$ as before.

Sketch of the proof. We use the Sobolev inequality that says that

$$
\left(\int u^{p} d x\right)^{2 / p} \leq C\|u\|_{H^{r}}^{2}
$$

for some $p>2$ depending on $r \in(0,1)$ and $N$; here $C$ depends also on $r$ and $N$. Actually, $p=2 N /(N-2 r)$ when $N \geq 2$ or when $N=1$ if, in addition, $0<r<1 / 2$. We want to control $\|u(t)\|_{q}$ for some $q>1$ using

$$
\int u^{q} d x \leq\left(\int u d x\right)^{\theta}\left(\int u^{p} d x\right)^{1-\theta}
$$

where $q=\theta+p(1-\theta)$. We will take the values

$$
\theta=\frac{p-2}{p}, \quad 1-\theta=\frac{2}{p} .
$$

The proof when $u \in L^{2}$ is quite similar.

The case $N=1$ with $1 / 2 \leq r<1$ is easy. Take $0<r^{\prime}<1 / 2$ and observe that $L^{1} \cap H^{r} \subset H^{r^{\prime}}$ as a continuous embedding. Now we can use (3.6), with $r^{\prime}$ replacing $r$, and get (3.4) with, perhaps, some different values of $q$ and $\theta$. 
Remark 3.3. We also recall that for every convex function $\Phi$ the quantity $\int \Phi(u(t)) d x$ is nonincreasing in time in the FPME evolution. We note that for such a function $\Phi$ we have

$$
\left.\int \Phi(u(x, t)) d x\right|_{T_{0}} ^{T_{1}}+\int_{T_{0}}^{T_{1}} \mathcal{B}(G(u), u) d t \leq 0,
$$

where $G(u)$ is the primitive of $\Phi^{\prime \prime}(u) u$. The bilinear form $\mathcal{B}$ is as above and

$$
\mathcal{B}(G(u), u) \geq c \mathcal{B}(u, u) \sim\|u\|_{H^{r}}^{2}
$$

if $G^{\prime}$ is strictly positive. We will use this in the case of truncations of the form $\Phi\left(u_{k}^{+}\right)$ with $u_{k}^{+}=(u-k)^{+}$and then $G=0$ for $u \leq k$.

\section{Boundedness of solutions}

This section is devoted to proving the main boundedness result, Theorem 1.1. In dimension $N=1$ this was proved in [3]. Our proof applies to all $N \geq 1$ and all $s \in(0,1)$, and is divided into three subsections.

\subsection{Better integrability properties for solutions}

We start with the following partial result.

Lemma 4.1 ("From $L^{1}$ to $\left.L \log L "\right)$. Let $u \geq 0$ be a weak energy solution of the FPME. If the initial data are integrable, then $u(\cdot, t) \in L \log L$ for all positive $t>0$, and for all small $t \leq t_{0}$ we have

$$
\int u(t) \log (1+u(t)) d x+\frac{1}{t} \int_{0}^{t} s \mathcal{B}\left(u(s)_{1}^{+}, u(s)_{1}^{+}\right) d s \leq C_{0}|\log (t)|\left\|u_{0}\right\|_{1},
$$

where $C_{0}$ depends only on $N, s$, and we write $u(t)_{1}^{+}=(u(t)-1)^{+}$. The time $t_{0}$ is estimated as $t_{0}=\inf \left\{1, C\left\|u_{0}\right\|_{L^{1}}^{-\vartheta}\right\}$ for some constants $C, \vartheta>0$.

Proof. We use as test function $\eta=t \log (1+u)$ in the weak form of the equation. After observing that $h(u)=(u+1) \log (1+u) \geq 0$ satisfies $\log (1+u)=h^{\prime}(u)-1$ and integrating over $\mathbb{R}^{N} \times(0, \tau)$, we get the identity

$$
\begin{aligned}
\tau \int h(u(\tau)) d x+ & \int_{0}^{\tau} t \mathcal{B}(g(u), u) d t \\
& =\int_{0}^{\tau} \int h(u(t)) d x d t+\tau \int u(\tau) d x-\int_{0}^{\tau} \int u d x d t
\end{aligned}
$$

where $\mathcal{B}=\mathcal{B}_{r}$ with $r=1-s$, as already explained, and $g^{\prime}(u)=u /(1+u)$ with $g(0)=0$. The last two terms in the display disappear by mass conservation. Note next 
that $g^{\prime}(u) \geq 1 / 2$ for $u \geq 1$, hence $g(u) \geq(1 / 2)(u-1)^{+}$, so that, writing $u_{1}^{+}=(u-1)^{+}$ we have, by the already stated monotonicity properties of $\mathcal{B}$,

$$
\mathcal{B}(g(u), u) \geq \frac{1}{2} \mathcal{B}\left(u_{1}^{+}, u\right) \geq \frac{1}{2} \mathcal{B}\left(u_{1}^{+}, u_{1}^{+}\right) .
$$

With this we arrive at

$$
\tau \int h(u(\tau)) d x+\frac{1}{2} \int_{0}^{\tau} t \mathcal{B}\left(u_{1}^{+}, u_{1}^{+}\right) d t \leq \int_{0}^{\tau} \int h(u(t)) d x d t .
$$

Recalling the definition of $h$ and using $\log (1+u) \leq u$, we get

$$
\begin{aligned}
\sup _{0<t<\tau} t \int u(t) \log (1+u(t)) d x+\frac{1}{2} & \int_{0}^{\tau} t \mathcal{B}\left(u_{1}^{+}, u_{1}^{+}\right) d t \\
& \leq \int_{0}^{\tau} \int u \log (u+1) d x d t+\tau \int u_{0} d x
\end{aligned}
$$

We still have a "bad term" on the right-hand side containing $\int u \log (1+u) d x$, and it is tackled as follows: note that the left-hand side controls $\int t d t \int u^{q} d x$ if $q$ is as in Lemma 3.2; take such a $q>2$. Then for any $M>2$ we have

$$
\int u \log (1+u) d x \leq \log (M+1) \int_{u<M} u d x+\frac{\log (1+M)}{M^{q-1}} \int_{u>M} u^{q} d x,
$$

where we have used the fact that $u \log (1+u) / u^{q}$ is decreasing in $u$ for $u \geq M$. Next, there is a constant $C(N, q)>0$ such that $u^{q} \leq C(u-1)_{+}^{q}$ for this range of $u$. Choose now $M=t^{-\alpha}$ and then $\beta<\alpha(q-1)-\varepsilon$ (taking care that $\left.\beta>1\right)$. With all this, we get, for small $\tau$,

$$
\begin{aligned}
& \int_{0}^{\tau} \int u \log (1+u) d x d t \\
& \leq C_{1} \int_{0}^{\tau} d t|\log (t)|\left(\sup _{t} \int u\right)+C_{2} \int_{0}^{\tau} t^{\beta} d t\left(\int(u-1)_{+}^{q} d x\right) \\
& \leq C_{1} \tau|\log (\tau)| \int u_{0} d x+C_{2} \int_{0}^{\tau} t^{\beta}\left\|u_{1}^{+}(t)\right\|_{L^{1}}^{\theta}\left\|u_{1}^{+}(t)\right\|_{H^{r}}^{2} d t
\end{aligned}
$$

Since $\beta>1$ the last term is controlled by the $\mathcal{B}$-energy term on the left-hand side of (4.3) for small $\tau$. In particular, we choose $0<\tau<1$ and $\tau^{\beta-1} \leq\left(2 C_{2}\left\|u_{0}\right\|_{L^{1}}^{\theta}\right)^{-1}$. The other term is a multiple of $\int u_{0}$, hence bounded. We get

$$
\sup _{0<t<\tau} t \int u(t) \log (1+u(t)) d x+\int_{0}^{\tau} t \mathcal{B}\left(u_{1}^{+}, u_{1}^{+}\right) d t \leq C_{3} \tau|\log (\tau)|\left\|u_{0}\right\|_{1} .
$$

Putting $t=\tau$ we get the result.

Lemma 4.2 ("From $L \log L$ to $L^{2}$ "). Initial data in the space $L \log L$ imply that $u(\cdot, t) \in L^{2}$ for all positive $t>0$ and the bound on the $L^{2}$ norm of $(u-1)^{+}$depends only on $t, s, N,\left\|u_{0}\right\|_{1}$, and $\left\|\left(u_{0}-1\right)^{+}\right\|_{L \log L}$. 
Proof. We define $v(x, t)=u(x, t) \vee 1$ so that $v=1+u_{1}^{+} \geq 1$ and $v_{t}=u_{t} \chi_{\{u>1\}}$. Recall the notation $u_{k}^{+}=(u-k)^{+}$. Then

$$
\frac{d}{d t} \int[v \log (v)-v] d x=\int \log (v) v_{t}=\int_{u>1} \log (v) u_{t} .
$$

Using the weak form of the equation with $\eta=\log v$ as test function, we get

$$
\left.\int[v \log (v)-v](t) d x\right|_{t_{1}} ^{t_{2}}+\int_{t_{1}}^{t_{2}} \int \nabla[\log (v)] u \nabla \mathcal{L} u d x d t=0 .
$$

We work out the last term for fixed time and observe that, since $u=v$ for $u>1$, we have

$$
\int_{u>1} \nabla[\log (v)] u \nabla \mathcal{L} u d x=\int \nabla v \cdot \nabla \mathcal{L} u d x=\int \nabla u_{1}^{+} \cdot \nabla \mathcal{L} u d x=\mathcal{B}_{r}\left(u_{1}^{+}, u\right) .
$$

Using again the monotonicity of $\mathcal{B}$ (see Corollary 3.1 and Remark 3.3), and putting $h(v)=v \log (v)-v$, we get

$$
\int h\left(v\left(t_{2}\right)\right) d x+\int_{t_{1}}^{t_{2}} \mathcal{B}_{r}\left(u_{1}^{+}, u_{1}^{+}\right) d t \leq \int h\left(v\left(t_{1}\right)\right) d x .
$$

Note that $h(v)$ is convex for $v>1$ and the right-hand side is bounded by a combination of $\left\|u_{0}\right\|_{1}$ and $\left\|\left(u_{0}-1\right)^{+}\right\|_{L} \log L$. Hence,

$$
\int_{0}^{t_{2}} \mathcal{B}_{r}\left(u_{1}^{+}, u_{1}^{+}\right) d t \leq C
$$

Recall finally that $\mathcal{B}_{r}\left(u_{1}^{+}, u_{1}^{+}\right) \sim\left\|u_{1}^{+}(\cdot, t)\right\|_{H^{r}}^{2}$ with $r=1-s$. Use Lemma 3.2 to conclude that $u_{1}^{+}(t) \in L^{q}\left(\mathbb{R}^{N}\right)$ for some $q>2$. More quantitatively, this together with (4.6) and Remark 3.3 gives the estimate

$$
\sup _{t>0} t\left\|u_{1}^{+}(t)\right\|_{L^{q}}^{q} \leq C,
$$

with $C$ depending as in the statement of the lemma. Interpolation with $L^{1}$ gives the result.

\subsection{Boundedness}

With the preceding results, we may assume that $u_{0} \in L^{1}\left(\mathbb{R}^{N}\right) \cap L^{2}\left(\mathbb{R}^{N}\right)$ after some displacement of the time origin. Then we can follow the De Giorgi approach (as outlined for instance in [9], [10]). We consider the truncations

$$
u_{j}^{+}(x, t)=\left(u-M\left(2-2^{-j}\right)\right)^{+}
$$

The value of the constant $M>0$ will be conveniently chosen later. Actually, we may assume $\int u_{0}^{2} d x$ is very small by selecting $M$ large. 
Claim. The following energy inequality holds for all $0<t_{1}<t_{2}$ :

$$
\int\left(u_{j}^{+}\left(t_{2}\right)\right)^{2} d x+2 M\left(2-2^{-j}\right) \int_{t_{1}}^{t_{2}} \mathcal{B}\left(u_{j}^{+}, u_{j}^{+}\right) d t \leq \int\left(u_{j}^{+}\left(t_{1}\right)\right)^{2} d x .
$$

To see this, we use the definition of weak solution for our FPME with $\eta=u_{j}^{+}$as a test function. Then, for $t_{1}<t_{2}$, we have

$$
\int_{t_{1}}^{t_{2}} \int u_{t} u_{j}^{+} d x d t=\frac{1}{2} \int\left(u_{j}^{+}\left(t_{2}\right)\right)^{2} d x-\frac{1}{2} \int\left(u_{j}^{+}\left(t_{1}\right)\right)^{2} d x .
$$

For the RHS we observe that $u=u_{j}^{+}+M\left(2-2^{-j}\right)$ whenever $u \geq M\left(2-2^{-j}\right)$. Hence,

$$
\begin{aligned}
\int_{u>M\left(2-2^{-j}\right)} u \nabla u_{j}^{+} & \cdot \nabla \mathcal{L} u d x=\frac{1}{2} \int\left(\nabla\left(u_{j}^{+}\right)\right)^{2} \cdot \nabla \mathcal{L} u d x+M\left(2-2^{-j}\right) \int \nabla u_{j}^{+} \cdot \nabla \mathcal{L} u d x \\
= & \frac{1}{2} \mathcal{B}\left(\left(u_{j}^{+}\right)^{2}, u\right)+M\left(2-2^{-j}\right) \mathcal{B}\left(u_{j}^{+}, u\right) \geq M\left(2-2^{-j}\right) \mathcal{B}\left(u_{j}^{+}, u_{j}^{+}\right) .
\end{aligned}
$$

In the last inequality we have used both Corollary 3.1 and Lemma 3.1. This gives (4.8).

We now fix $t_{0}>0$. We want to prove that the solution $u$ is bounded for all times $t \geq t_{0}$. As in [10], let us define the total energy for the truncated function $u_{j}^{+}$as

$$
\mathcal{A}_{j}=\sup _{t \geq T_{j}} \int\left(u_{j}^{+}(t)\right)^{2} d x+2 M \int_{T_{j}}^{\infty} \mathcal{B}\left(u_{j}^{+}, u_{j}^{+}\right) d t
$$

where $T_{j}=t_{0}\left(1-2^{-j}\right)$. From (4.8), taking arbitrary values $t_{2}=t \geq T_{j}$ and $t_{1}=t^{\prime} \in$ $\left[T_{j-1}, T_{j}\right]$ we have

$$
\mathcal{A}_{j} \leq \inf _{t^{\prime} \in\left[T_{j-1}, T_{j}\right]} \int\left(u_{j}^{+}\left(t^{\prime}\right)\right)^{2} d x
$$

Observe now that $u_{j}^{+}(x)>0$ implies $u_{j-1}^{+}(x)=u_{j}^{+}(x)+M 2^{-j}>M 2^{-j}$. Therefore, for every $p>2$ we have (keeping the time fixed)

$$
\begin{aligned}
\int\left(u_{j}^{+}\right)^{2} d x & =\int\left(u_{j-1}^{+}-M 2^{-j}\right)^{2} \cdot \chi_{\left\{u_{j}^{+}>0\right\}} d x \\
& \leq \int\left(u_{j-1}^{+}\right)^{2}\left(\frac{u_{j-1}^{+}}{2^{-j} M}\right)^{p-2} d x=\left(\frac{2^{j}}{M}\right)^{p-2} \int\left(u_{j-1}^{+}\right)^{p} d x .
\end{aligned}
$$

If $p>2$ is the exponent corresponding to Sobolev's embedding theorem, we deduce from (4.9) that

$$
\begin{aligned}
\mathcal{A}_{j} & \leq \inf _{t^{\prime} \in\left[T_{j-1}, T_{j}\right]}\left(\frac{2^{j}}{M}\right)^{p-2} \int\left(u_{j-1}^{+}\left(t^{\prime}\right)\right)^{p} d x \\
& \leq C_{N}\left(2^{j} / M\right)^{p-2} \inf _{t^{\prime} \in\left[T_{j-1}, T_{j}\right]}\left[\mathcal{B}\left(u_{j-1}^{+}, u_{j-1}^{+}\right)\left(t^{\prime}\right)\right]^{p / 2} .
\end{aligned}
$$


Taking averages in $t^{\prime}$ we arrive at the inequality

$$
\begin{aligned}
\mathcal{A}_{j} & \leq C_{N}\left(2^{j} / M\right)^{p-2}\left[\frac{1}{T_{j}-T_{j-1}} \int_{T_{j-1}}^{T_{j}} \mathcal{B}\left(u_{j-1}^{+}, u_{j-1}^{+}\right)\left(t^{\prime}\right) d t^{\prime}\right]^{p / 2} \\
& \leq C_{N} \frac{2^{j(p-2)} 2^{j p / 2}}{M^{p-2}\left(M t_{0}\right)^{p / 2}}\left[M \int_{T_{j-1}}^{\infty} \mathcal{B}\left(u_{j-1}^{+}, u_{j-1}^{+}\right)\left(t^{\prime}\right) d t^{\prime}\right]^{p / 2} .
\end{aligned}
$$

This leads to a recurrence relation of the form

$$
\mathcal{A}_{j} \leq C^{j}\left(\frac{\mathcal{A}_{j-1}}{M t_{0}}\right)^{1+\delta}
$$

with $\delta=p / 2-1>0$, which implies $\mathcal{A}_{\infty}=0$ if $\mathcal{A}_{0} /\left(M t_{0}\right)$ is small. This determines the correct value of $M$ to choose. The conclusion is then that $u(x, t) \leq 2 M$ for all $t \geq t_{0}$.

\subsection{End of proof of the theorem}

The preceding subsections have established the result for any fixed $t>0$, and we know that

$$
u(x, t) \leq C\left(N, s,\left\|u_{0}\right\|_{1}, t\right)
$$

but we do not know the precise dependence of $C$ on its arguments. We need to prove that this dependence takes the form (1.5). This is just a consequence of the scaling group that allows us to pass from a solution $u(x, t)$ to the rescaled solution

$$
\widetilde{u}(x, t)=A u(L x, T t)
$$

on the condition that $A=T L^{-2+2 s}$. On the other hand, we want to reduce $\tilde{u}$ to unit mass, $\int \tilde{u}(x, t) d x=1$, and this means $A=L^{N} /\left\|u_{0}\right\|_{1}$. Altogether this gives (with $\left.\left\|u_{0}\right\|_{1}=M_{1}\right)$

$$
L=\left(M_{1} T\right)^{\beta}, \quad A=M_{1}^{2(1-s) \beta} T^{-N \beta},
$$

where $\beta=1 /(N+2-2 s)$. We now apply the boundedness result to $\tilde{u}$ at $t=1$, i.e.,

$$
\widetilde{u}(x, 1) \leq C(N, s) \quad \forall x \in \mathbb{R}^{N} .
$$

Going back to $u$, we have

$$
\sup _{x} u(x, T)=A \sup _{x} \tilde{u}(x, 1)=M_{1}^{2(1-s) \beta} T^{-N \beta} C(N, s),
$$

which gives the desired result upon replacing $T$ by $t$. For other instances of this scaling argument cf. [21]. 


\section{Hölder regularity. Main result and basic lemmas}

Once the question of boundedness is settled, we proceed with the local regularity of solutions and prove our main result, Theorem 1.2.

Strategy. Since the equation is space- and time-invariant we may assume that $T_{1}<$ $T_{2}=0$, and then we may study the regularity around $x=0$ and $t=0$.

The main ideas are two: on the one hand, we will prove some basic De Giorgi-type oscillation lemmas that say that the oscillation of the solution $u$ decays when we restrict a basic domain, say, the cylinder $\Gamma_{4}=B_{4}(0) \times[-4,0]$, to a smaller cylinder like $\Gamma_{1}=$ $B_{1}(0) \times[-1,0]$. The second ingredient is the scaling property of the equation that allows one to renormalize the solution through the transformation

$$
\widehat{u}(x, t)=A u(B x, C t)
$$

with $C, B>0$ free parameters, and $A=C B^{-2+2 s}$. The way of attacking the problem is through the iterated application of the lemmas. At the end of every step we renormalize the solution defined in $\Gamma_{1}$ to a rescaled solution defined in $\Gamma_{4}$ and we start a new application of the oscillation lemmas. In this way, we will show that the oscillation of the solution $u$ decays dyadically in a family of space-time cylinders shrinking dyadically to a point.

The needed lemmas have a simpler expression for $0<s<1 / 2$ where the diffusion is more similar to the standard porous medium case. For $1 / 2 \leq s<1$ convection effects appear that make some integrals diverge, and this makes the analysis more difficult, needing new techniques. The detailed study of how to proceed in the case $s>1 / 2$ is contained in Section 12. Until then we assume that $s<1 / 2$.

\subsection{The oscillation reduction lemmas}

These technical results need only be proved for bounded nonnegative weak solutions defined in a strip $S_{R}=\mathbb{R}^{N} \times[-R, 0]$. We denote by $\Gamma_{R}$ the parabolic cylinder $B_{R}(0) \times[-R, 0]$. One of the lemmas controls the decrease of the supremum of the solution once we restrict the size of the parabolic neighbourhood of $(0,0)$, the other one implies that under suitable assumptions the solution separates from zero. A third one improves the first result so as to obtain a real alternative between going a bit down and a bit up, which leads to the proof of regularity.

Here is the first basic lemma.

Lemma 5.1. Let $0<s<1 / 2$. Given $\mu \in(0,1 / 2)$ and $\varepsilon_{0}$ small enough (in particular, $\varepsilon_{0} \leq 1-2 s$ ), there exists $\delta>0$ (depending possibly on $\mu, \varepsilon_{0}, s$, and $N$ ) such that if we assume that

(i) the solution $u$ is bounded above in the strip $S_{4}=\mathbb{R}^{N} \times[-4,0]$ by

$$
\bar{\Psi}(x)=1+\left(|x|^{\varepsilon}-2\right)^{+}, \quad 0<\varepsilon<\varepsilon_{0},
$$


(ii) $u$ is mostly below the level $1 / 2$ in $\Gamma_{4}=B_{4}(0) \times[-4,0]$ in the sense that

$$
\left|\{u>1 / 2\} \cap \Gamma_{4}\right| \leq \delta\left|\Gamma_{4}\right|,
$$

then we can lower the upper bound inside a smaller cylinder in the following quantitative form: $\left.u\right|_{\Gamma_{1}} \leq 1-\mu$.

We summarize the result by saying that "being mostly below $1 / 2$ in space-time measure pulls down the supremum in a smaller nested cylinder". Note that for this lemma, $\delta$ can be chosen as a nonincreasing function of $\mu$ with $\delta(1 / 2+)=0$. We also remark that the size of the cylinders can be changed, though this affects the values of $\delta$ if the new sizes do not conform with the parabolic scaling. Finally, the levels $u=1 / 2$ and $u=1$ are taken by convenience, any pair of levels $0<M_{1}<M_{2}$ will do, though in principle the value of $\delta$ will change.

A similar result applies from below but the proof is different since the equation is degenerate at $u=0$. The idea is that if $u$ is very often far from zero in $\Gamma_{4}$ then in a smaller, suitably nested cylinder, $u$ stays uniformly away from zero. The technical version explains how "if on most of the space-time the solution is above $1 / 2$, it pulls up away from zero".

Lemma 5.2. Under the same assumptions, given $\mu_{0} \in(0,1 / 2)$ there exists $\delta>0$ (depending possibly on $\mu_{0}, \varepsilon_{0}, s$, and $N$ ) such that if $u$ satisfies

$$
\left|\{u \geq 1 / 2\} \cap \Gamma_{4}\right| \geq(1-\delta)\left|\Gamma_{4}\right|,
$$

then $\left.u\right|_{\Gamma_{1}} \geq \mu_{0}$.

Again, $\delta$ is a nonincreasing function of $\mu_{0}$. Let $\delta_{0}=\delta(1 / 4)$, that is, when $\mu_{0}=1 / 4$. A more elaborate version of this lemma will be needed in some cases of the iteration.

We complement these two lemmas with a lemma that replaces the "most of the spacetime" assertion of Lemma 5.1 by "in some set of positive measure".

Lemma 5.3 ("Some of the space-time below, pulls down"). Assume as before that $0<$ $s<1 / 2$ and $u$ is trapped between 0 and $\bar{\Psi}$ in $S_{4}$. Moreover, assume now that

$$
\left|\{u<1 / 2\} \cap \Gamma_{4}\right| \geq \delta_{0}\left|\Gamma_{4}\right|,
$$

with $\delta_{0}$ defined as above. Then $\left.u\right|_{\Gamma_{1}} \leq 1-\mu^{\prime}$ for some $\mu^{\prime}\left(\delta_{0}\right)$.

Notice that this last lemma applies only in one direction, reducing the oscillation from above. As in the classical porous media, we cannot expect this lemma to hold in the "pulling-up" case, due to the property of finite propagation (existence of solutions with compact support), a consequence of the degeneration of the equation. Nevertheless, this one-sided improvement will be enough to prove that the oscillation decays dyadically as follows:

The iterated use of Lemma 5.3 from above after rescaling at every step, as long as possible, reduces the oscillation of $u$ from above and we start iterating and renormalizing 
to get $u_{2}, u_{3}, \ldots$ We note though that, as we do that, a renormalized solution develops a "tail" in the sense that the functions $u_{k}$ start to grow at infinity by effect of the scaling (both in vertical and horizontal directions). This is the reason for the form of the upper barrier that we use, which has an $\varepsilon$-tail. Indeed, after $k$ steps, $u$ will be bounded by $(1-\mu)^{-k}$ outside the $B^{-k}$ dilation using (5.1). This first difficulty can be dealt with by playing the integrability of the kernels $L$ or $K$ at infinity against a slow power growth in $u$. Indeed, by sacrificing the modulus of Hölder continuity we may assume that the gain is very tiny (i.e., $\mu$ is very small). Then the build up in $u(y, t)$ as $y$ tends to infinity will be very slow (like $|y|^{\varepsilon}$ ), being absorbed by $K$. If Lemma 5.3 never fails along the iteration, then we are at a point where $u=0$ and a Hölder exponent is also found.

On the other hand, if the process breaks down, then the first time Lemma 5.3 fails, it puts us under the hypotheses of Lemma 5.2 and that pulls $u$ away from zero by a fixed amount $\mu_{0}$ (the need for an alternative to Lemma 5.3 is what makes Lemma 5.1 insufficient). Then the operator becomes nondegenerate in the subsequent iterations and a counterpart of Lemma 5.3 applies also from below (upwards) since $u$ will always be bounded between $\mu_{0}$ and 1 . From there on the gain in the oscillation of $u$ may come from above or below in the dyadic way we have shown. The details of the iteration are given in Section 11 after the lengthy and delicate proof of the lemmas is completed. We recall that all this will be done below for $0<s<1 / 2$.

\section{Lowering the maximum. Proof of Lemma 5.1}

We start here the technical work. The basic idea in the proof of the result is a particular kind of "localized energy inequalities" that will be iterated in the style of De Giorgi to obtain the reduction of the maximum in a smaller domain. Localization is obtained by using a suitable sequence of cutoff functions. In order to deduce the necessary energy inequalities we use integration by parts formulas and analysis of the kernels. A main role is played by the bilinear form $\mathcal{B}(v, w)$ as defined in (3.2) with kernel $K(x)=c|x|^{-(N+2 r)}$ and $r=1-s$. Moreover, we put $L(x)=c_{1}|x|^{-N+2 s}$ so that $-\Delta L=K$. We will repeatedly use the equivalent form justified by Proposition 3.2, which reads here

$$
\mathcal{B}(u, v)=C \iint(u(x)-u(y)) K(x-y)(v(y)-v(x)) d x d y .
$$

We take a weak energy solution defined in a strip $(-T, 0) \times \mathbb{R}^{N}$ in the sense of Section 2 . We justify the computations by recalling that $u$ can be approximated by smooth positive solutions of similar problems as done in [11].

\subsection{An energy formula}

We consider a sequence of cutoffs $\varphi(x)$ that have the form of perturbations of the level $u=1$ within a region containing the unit ball $B_{1}(0)$, and an "outer wing" rising up above the 1-level for larger values of $|x|$. An explicit choice suiting our purposes will be 
specified below. We only need to know at this stage that the cutoff function $\varphi$ is smooth, lies above $1 / 2$ everywhere, and also that $u \leq \varphi$ for all $|x| \geq 3$ for all times $-4 \leq t \leq 0$.

We use the function $\eta=\log ((u / \varphi) \vee 1)=\log g$ as a test function in the weak form of the equation. Note that

$$
g:=\frac{u}{\varphi} \vee 1=1+\frac{(u-\varphi)^{+}}{\varphi}=1+\frac{u_{\varphi}^{+}}{\varphi},
$$

where $u_{\varphi}^{+}=(u-\varphi)^{+}$according to the adopted notation. Note that $g \geq 1$, and $g>1$ iff $u>\varphi$. According to our assumptions, $u_{\varphi}^{+}$and $g-1$ have compact support in the ball of radius 3 . We will often split $u$ as

$$
u=u_{\varphi}^{+}+\varphi+(u-\varphi)^{-}
$$

where we write $(u-\varphi)^{-}=(u-\varphi) \wedge 0=u_{\varphi}^{-}$. Notice that with this notation we have $u_{\varphi}^{-} \leq 0$. After applying the weak formulation of the equation with $\eta$ as above, we get on the LHS, for $T_{1} \leq t \leq T_{2} \leq 0$,

$$
\begin{gathered}
\int_{T_{1}}^{T_{2}} \int \eta \partial_{t} u d x d t=\int_{T_{1}}^{T_{2}} \int \log \left(\frac{u}{\varphi} \vee 1\right) \varphi \partial_{t}(u / \varphi) d x d t \\
=\left.\int \varphi\left(\frac{u}{\varphi} \vee 1\right)\left[\log \left(\frac{u}{\varphi} \vee 1\right)-1\right] d x\right|_{T_{1}} ^{T_{2}}=\left.\int \varphi(g \log g-g) d x\right|_{T_{1}} ^{T_{2}}
\end{gathered}
$$

We will need an estimate of this quantity: after adding 1 to the last integrand we get the expression $H(g):=g \log g+1-g$ for which we have the estimate $H(g) \sim(g-1)^{2}$ for $1 \leq g \leq 2$, in the sense that

$$
\frac{1}{2}\left(\frac{u_{\varphi}^{+}}{\varphi}\right)^{2} \leq H(g) \leq\left(\frac{u_{\varphi}^{+}}{\varphi}\right)^{2}
$$

Let us now calculate the right-hand side of the expression in the weak formulation of the equation. We have

$$
\begin{aligned}
& \int d t \int \log (g(x)) \operatorname{div}[u(x) \nabla L(x-y)(u(y)-u(x))] d x d y \\
&=-\int d t \int_{u>\varphi} \frac{\nabla g(x)}{g(x)} u(x) \nabla_{x} L(x-y)(u(y)-u(x)) d x d y=\mathbf{I}+\mathbf{I I}
\end{aligned}
$$

where we pass from the first line to the second integrating by parts. Recalling that

$$
g=\frac{u}{\varphi} \vee 1=1+\frac{u_{\varphi}^{+}}{\varphi}, \quad \nabla g=\nabla\left(u_{\varphi}^{+} / \varphi\right) \chi_{\{u>\varphi\}},
$$


the first part of the splitting is

$$
\begin{aligned}
\mathbf{I} & =-\int d t \iint u_{\varphi}^{+}(x)(-\Delta L)(x-y)[u(y)-u(x)] d x d y \\
& =-\int d t \iint u_{\varphi}^{+}(x) K(x-y)[u(x)-u(y)] d x d y .
\end{aligned}
$$

After symmetrizing, we get $\mathbf{I}=-\frac{1}{2} \int \mathcal{B}\left(u_{\varphi}^{+}, u\right) d t$, where

$$
\mathcal{B}\left(u_{\varphi}^{+}, u\right):=\iint\left(u_{\varphi}^{+}(x)-u_{\varphi}^{+}(y)\right) K(x-y)(u(x)-u(y)) d x d y .
$$

On the other hand,

$$
\mathbf{I I}=\int d t \iint u_{\varphi}^{+}(x) \frac{\nabla \varphi(x)}{\varphi(x)} \nabla_{x} L(x-y)[u(y)-u(x)] d x d y=: \int \mathcal{Q}\left(u_{\varphi}^{+}, u\right) d t .
$$

- In order to separate the good and bad components of both $\mathcal{B}$ and $\mathcal{Q}$, we use the decomposition $u=u_{\varphi}^{+}+\varphi+u_{\varphi}^{-}$. We get

$$
\mathcal{B}\left(u_{\varphi}^{+}, u\right)=\mathcal{B}\left(u_{\varphi}^{+}, u_{\varphi}^{+}\right)+\mathcal{B}\left(u_{\varphi}^{+}, \varphi\right)+\mathcal{B}\left(u_{\varphi}^{+}, u_{\varphi}^{-}\right) .
$$

and a similar expression for $\mathcal{Q}$. We now make some observations:

(i) $\mathcal{B}\left(u_{\varphi}^{+}, u_{\varphi}^{+}\right)$is a positive quadratic form. We will pass the corresponding part of $\mathbf{I}$ to the LHS as a term with positive sign and thus complete the energy expression in the energy inequality that we want to derive.

(ii) $\mathcal{B}\left(u_{\varphi}^{+}, u_{\varphi}^{-}\right)$also has the correct nonnegative sign because of these facts: $u_{\varphi}^{+}$and $u_{\varphi}^{-}$ have opposite signs and disjoint supports, and $K \geq 0$. We could drop this term in a first calculation, but we will keep it and use it to control some of the bad terms in $\mathcal{Q}$.

Summing up, we have up to now the basic identity for $T_{1}<T_{2} \leq 0$ :

$$
\begin{gathered}
\left.\int \varphi(g \log g+1-g)\right|_{T_{2}} d x+\frac{1}{2} \int_{T_{1}}^{T_{2}} \mathcal{B}\left(u_{\varphi}^{+}, u_{\varphi}^{+}\right) d t+\frac{1}{2} \int_{T_{1}}^{T_{2}} \mathcal{B}\left(u_{\varphi}^{+}, u_{\varphi}^{-}\right) d t \\
=\left.\int \varphi(g \log g+1-g)\right|_{T_{1}} d x-\frac{1}{2} \int_{T_{1}}^{T_{2}} \mathcal{B}\left(u_{\varphi}^{+}, \varphi\right) d t+\int_{T_{1}}^{T_{2}} \mathcal{Q}\left(u_{\varphi}^{+}, u\right) d t .
\end{gathered}
$$

(iii) We will think of the LHS as the basic energy of this calculation, and the RHS as the terms still to be controlled. 


\subsection{Cutoff functions, control of the RHS and final goal}

In order to tackle the RHS and continue the proof of the lemma, we need to make a convenient choice of the sequence of cutoffs. Though only some simple bounds on the functions and their derivatives are used, a possible practical choice is as follows:

$$
\varphi_{k}(x)=\min \left\{1+\left(|x|^{\varepsilon}-2\right)^{+}, \bar{\varphi}_{k}(x)\right\}, \quad \bar{\varphi}_{k}(x)=\frac{7}{8}+\frac{|x|^{2}}{16}-\frac{1}{2} 4^{-k},
$$

for some small $\varepsilon>0$ and $k=1,2, \ldots$.

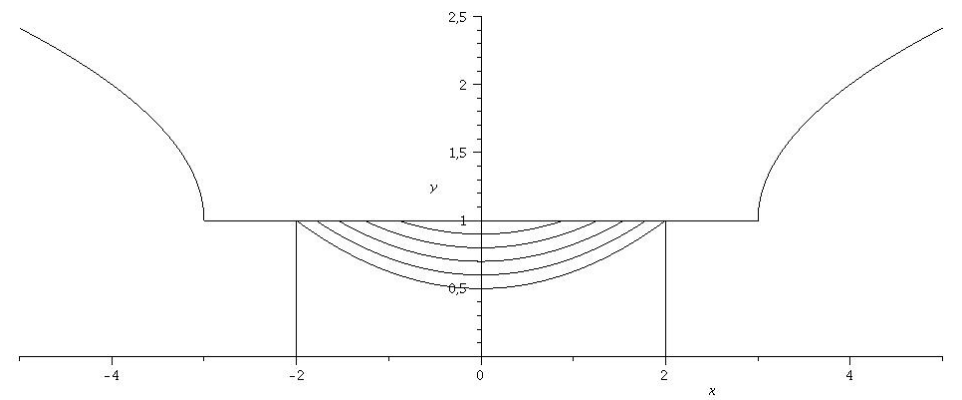

Note that $\varphi_{k} \geq \varphi_{k-1}$. The following remark will be important: at points where $\varphi_{k}<1$ we have

We also have

$$
\varphi_{k}=\varphi_{k-1}+\frac{1}{2} 4^{-k}
$$

$$
\inf \varphi_{k}=\varphi_{k}(0)>1 / 2 \quad \text { for } k \geq 1 .
$$

Moreover, $\varphi_{\infty}(x) \leq 1$ precisely for $|x| \leq \sqrt{2}$, and $\varphi_{1}(x)<1$ for $|x|<2$. This means in particular that $\varphi_{k}(x)=1+\left(|x|^{\varepsilon}-2\right)^{+}$for $|x| \geq 2, k \geq 1$. Moreover, $\varphi_{\infty}(x)=$ $\left(|x|^{2}+14\right) / 16 \leq 15 / 16$ for $|x| \leq 1$.

A more general version of the same construction takes

$$
\bar{\varphi}_{k}(x)=1-\frac{1}{2 C}+\frac{|x|^{2}}{4 C}-\frac{1}{2} C^{-k}
$$

with $C$ possibly larger than 4 . In that case $1-\varphi_{\infty}(x) \geq 1 / 4 C$ for $|x| \leq 1$.

For the rest of this proof we write $u_{k}^{+}=\left(u-\varphi_{k}\right)^{+} \geq 0, u_{k}^{-}=\left(u-\varphi_{k}\right)^{-} \leq 0$. Notice that the support of $u_{k}^{+}$is contained in the ball of radius 2 as a consequence of (5.2).

We are ready to tackle the RHS of (6.5) with this choice of test functions. One part will be controlled by a small multiple of the present energy, i.e., we will absorb it into the LHS of (6.5). The rest will be bounded above by a large multiple of $\left|\left\{u_{k}^{+}>0\right\}\right|$ (the notation $|\cdot|$ means the measure of the set). We recall our goal: if we do this, together with the Sobolev inequality, we will get an iterative relation for the LHS energies

$$
\mathcal{A}_{k+1} \leq C^{k}\left(\mathcal{A}_{k}\right)^{1+\sigma}
$$

that converges to zero as $k \rightarrow \infty$, as desired, if the iteration is started from a small initial value $\mathcal{A}_{0}$. 


\subsection{Estimate of the remaining $\mathcal{B}$ term}

We start the process with $\mathcal{B}_{2}=\mathcal{B}\left(u_{k}^{+}, \varphi_{k}\right)$. By inspecting the integral we easily get

$$
\mathcal{B}_{2} \leq \gamma \mathcal{B}\left(u_{k}^{+}, u_{k}^{+}\right)+\frac{1}{\gamma} \mathcal{B}^{*}\left(\varphi_{k}, \varphi_{k}\right)
$$

for every $\gamma>0$, where $\mathcal{B}^{*}\left(\varphi_{k}, \varphi_{k}\right)$ indicates that the integral is performed only on the set where either $x$ or $y$ belongs to $\left\{u_{k}^{+}>0\right\}$. That is,

$$
\mathcal{B}^{*}=\iint\left[\chi_{\left\{u_{k}^{+}>0\right\}}(x)+\chi_{\left\{u_{k}^{+}>0\right\}}(y)\right] K(x-y)\left(\varphi_{k}(x)-\varphi_{k}(y)\right)^{2} d x d y .
$$

For $\gamma$ small, $\gamma \mathcal{B}\left(u_{k}^{+}, u_{k}^{+}\right)$is then absorbed into the LHS (into the energy). Now, using

$$
\left|\varphi_{k}(x)-\varphi_{k}(y)\right| \leq C \min (1,|x-y|),
$$

and the size of the kernel $K$, we arrive at the estimate

$$
\mathcal{B}^{*} \leq C\left|\left\{u_{k}^{+}>0\right\}\right| \leq C 4^{2 k} \int_{u_{k}^{+}>0}\left(u_{k-1}^{+}\right)^{2} d x .
$$

The last inequality follows by Chebyshev's inequality, since $u_{k-1} \geq 4^{-k} / 2$ whenever $u_{k}^{+}>0$. The expression obtained is good for our later purposes.

\subsection{Analysis of the $\mathcal{Q}$ terms for $0<s<1 / 2$}

The last term in (6.5) also has a bilinear structure. Indeed,

$$
\begin{aligned}
\iint u_{k}^{+}(x) \frac{\nabla \varphi_{k}(x)}{\varphi_{k}(x)} & \nabla L(x-y)[u(x)-u(y)] d x d y=: \mathcal{Q}\left(u_{k}^{+}, u\right) \\
& =\mathcal{Q}\left(u_{k}^{+}, u_{k}^{+}\right)+\mathcal{Q}\left(u_{k}^{+}, \varphi_{k}\right)+\mathcal{Q}\left(u_{k}^{+}, u_{k}^{-}\right)=\mathcal{Q}_{1}+\mathcal{Q}_{2}+\mathcal{Q}_{3},
\end{aligned}
$$

but note that the "kernel" that is involved is not symmetric due to the presence of terms with $\varphi_{k}$. The study of the contribution of each of the three terms is again split into the close-range and far-field interactions, represented by the integrals for $x-y$ lying in a ball around the origin, or in its complement. In that sense we note that $\nabla L$ satisfies $|\nabla L| \leq$ $c|x-y| K(x, y)$. This will be used repeatedly.

- Let us first tackle the integral $\mathcal{Q}_{1}$ in a ball of radius $\eta$ around the origin:

$$
\begin{aligned}
\left|\mathcal{Q}\left(u_{k}^{+}, u_{k}^{+}\right)^{\mathrm{int}}\right| \leq & \iint_{|x-y| \leq \eta} u_{k}^{+}(x)\left|\frac{\nabla \varphi_{k}(x)}{\varphi_{k}(x)}\right| c|x-y| K(x, y)\left|u_{k}^{+}(x)-u_{k}^{+}(y)\right| d x d y \\
\leq & 8 c^{2} \iint_{|x-y| \leq \eta} u_{k}^{+}(x)^{2}\left|\nabla \varphi_{k} / \varphi_{k}\right|^{2}|x-y|^{2} K(x, y) d x d y \\
& +\frac{1}{4} \iint_{|x-y| \leq \eta} K(x, y)\left|u_{k}^{+}(x)-u_{k}^{+}(y)\right|^{2} d x d y .
\end{aligned}
$$


The last integral is just the part of $\frac{1}{4} \mathcal{B}\left(u_{k}^{+}, u_{k}^{+}\right)$integrated for $|x-y| \leq \eta$, so it can be absorbed by the LHS energy. The first integral is first integrated in $y$, which is easy since

$$
\int_{|x-y| \leq \eta} K(x, y)|x-y|^{2} d y=O\left(\eta^{2 s}\right) .
$$

Using this and also $|\nabla \varphi / \varphi| \leq C$, this first integral can then be estimated as

$$
\leq C \eta^{2 s} \int\left(u_{k}^{+}\right)^{2} d x
$$

an expression that can be left on the RHS or absorbed into the LHS if we take $\eta$ small.

- Let us compute the outer part of $\mathcal{Q}_{1}$ (for $|x-y|>\eta$ ). In this region, $\nabla L$ is integrable, so that

$$
\left|\mathcal{Q}\left(u_{k}^{+}, u_{k}^{+}\right)^{\text {out }}\right| \leq C(\eta)\left(\int\left(u_{k}^{+}\right)^{2} d x+\left(\int u_{k}^{+} d x\right)^{2}\right),
$$

which is also admissible, as we will see. Note that the last integral comes from the term in $u_{k}^{+}(x) u_{k}^{+}(y)$.

- Next, we treat the term

$$
\mathcal{Q}_{2}=\mathcal{Q}\left(u_{k}^{+}, \varphi_{k}\right)=\iint u_{k}^{+} \frac{\nabla \varphi_{k}}{\varphi_{k}} \nabla L(x-y)\left[\varphi_{k}(x)-\varphi_{k}(y)\right] d x d y .
$$

Remember that $u_{k}^{+}(x)$ is compactly supported in a small ball. For $|x-y| \leq 4$ we have $\left|\varphi_{k}(x)-\varphi_{k}(y)\right| \leq C|x-y|$, so

$$
\nabla L(x-y)\left[\varphi_{k}(x)-\varphi_{k}(y)\right]
$$

is integrable in $y$ and we are left with

$$
C \int u_{k}^{+}(x) d x \leq C\left|\left\{u_{k}^{+}>0\right\}\right| \leq C^{k} \int_{u_{k}^{+}>0}\left(u_{k-1}^{+}\right)^{2} d x
$$

which is also a good term.

- For $|x-y|$ larger the calculation is more involved. We will use the fact that $\nabla L$ has mean value zero on spheres. Therefore, we write $\varphi_{k}(x)-\varphi_{k}(y)=\left(\varphi_{k}(x)-\varphi_{k}(y-x)\right)+$ $\left(\varphi_{k}(y-x)-\varphi_{k}(y)\right)$. We observe that

$$
\int \nabla L(x-y)\left(\varphi_{k}(x)-\varphi_{k}(y-x)\right) d y=\int_{|z| \geq 4} \nabla L(z)\left(\varphi_{k}(x)-\varphi_{k}(z)\right) d z
$$

is zero in the sense of principal value since (i) $\varphi_{k}(x)-\varphi_{k}(z)$ is a radial function of $z$, (ii) we have an antisymmetry property for $\nabla L$; both facts imply the cancellation of the integral. The rest of the integral is

$$
\int \nabla L(x-y)\left(\varphi_{k}(y-x)-\varphi_{k}(y)\right) d y .
$$


Since $|\nabla L(x-y)| \sim|x-y|^{-(N+1-2 s)}$ as $|y| \rightarrow \infty$ and $\left|\varphi_{k}(y-x)-\varphi_{k}(x)\right| \sim C|x||y|^{\varepsilon-1}$ the whole integral is convergent if $2>2 s+\varepsilon$, which is a smallness condition on $\varepsilon_{0}$. Performing then the integration in $x$, we conclude that

$$
\left|\mathcal{Q}\left(u_{k}^{+}, \varphi_{k}\right)\right| \leq C\left|\left\{u_{k}^{+}>0\right\}\right| \leq C^{k} \int_{u_{k}^{+}>0}\left(u_{k-1}^{+}\right)^{2} d x
$$

as desired.

- The last term to examine is

$$
\mathcal{Q}_{3}=\mathcal{Q}\left(u_{k}^{+}, u_{k}^{-}\right)=-\iint u_{k}^{+}(x) \frac{\nabla \varphi(x)}{\varphi(x)} \nabla L(x-y) u_{k}^{-}(y) d x d y .
$$

For $|x-y| \leq \eta$ small, we use $|\nabla L(x-y)| \leq C|x-y| K(x-y)$ and then $Q\left(u_{k}^{+}, u_{k}^{-}\right)$ is bounded by a small fraction of $\mathcal{B}\left(u_{k}^{+}, u_{k}^{-}\right)$(remember that this term had the right sign). We can therefore get this part absorbed by the LHS of the energy identity.

- Finally, for $|x-y|>\eta$, we have the worst convergence case. This is the only place where we use the restriction $s<1 / 2$. We solve the difficulty of the integrability in $y$ at infinity by taking $\varepsilon<\varepsilon_{0}=1-2 s$, so that integration first in $y$ is bounded, since the term $\left|\nabla L(x-y) u_{k}^{-}(y)\right|$ is of the form $O\left(|y|^{2 s+\varepsilon-N-1}\right)$. We conclude that

$$
\left|\mathcal{Q}\left(u_{k}^{+}, u_{k}^{-}\right)^{\text {out }}\right| \leq C\left|\left\{u_{k}^{+}>0\right\}\right| \leq C^{k} \int_{u_{k}^{+}>0}\left(u_{k-1}^{+}\right)^{2} .
$$

Summary. Using (6.3), we obtain for $0<s<1 / 2$ and $t_{1}<t_{2} \leq 0$ the following energy inequality:

$$
\begin{aligned}
\int \frac{\left(u_{k}^{+}\left(t_{2}\right)\right)^{2}}{\varphi_{k}} d x+\frac{1}{2} \int_{t_{1}}^{t_{2}} \mathcal{B}\left(u_{k}^{+}, u_{k}^{+}\right) d t \\
\quad \leq 2 \int \frac{\left(u_{k}^{+}\left(t_{1}\right)\right)^{2}}{\varphi_{k}} d x+C^{2 k} \int_{t_{1}}^{t_{2}} \int_{u_{k}^{+}>0}\left(u_{k-1}^{+}\right)^{2} d x d t,
\end{aligned}
$$

where $C$ is a universal constant that only depends on $s$ and the dimension, $N$. In the application to the iteration, the $t_{i}$ will be chosen in dependence on $k$.

\subsection{Iteration and end of proof of Lemma 5.1}

This part is very similar to the one at the end of the boundedness proof in Section 4.2. We define the total energy function for the truncated solution $u_{k}^{+}$as

$$
\mathcal{A}_{k}=\sup _{T_{k} \leq t \leq 0} \int\left(u_{k}^{+}(t)\right)^{2} d x+\int_{T_{k}}^{0} \mathcal{B}\left(u_{k}^{+}, u_{k}^{+}\right) d t
$$


where $T_{k}=-2\left(1+2^{-k}\right), k=0,1, \ldots$ Notice that $\varphi_{k}$ lies between $1 / 2$ and 1 at the points where $u_{k}^{+}$is not zero. From (6.10) with $k \geq 1$, taking arbitrary values $t_{2}=t \geq T_{k}$ and $t_{1}=t^{\prime} \in\left[T_{k-1}, T_{k}\right]$ we have

$$
\mathcal{A}_{k} \leq 4 \inf _{t^{\prime} \in\left[T_{k-1}, T_{k}\right]} \int\left(u_{k}^{+}\left(t^{\prime}\right)\right)^{2} d x+C^{2 k} \int_{t^{\prime}}^{0} \int_{u_{k}^{+}>0}\left(u_{k-1}^{+}\right)^{2} d x d t=I+I I .
$$

Taking averages in $t^{\prime}$ we arrive at the inequality

$$
\begin{aligned}
\inf _{t^{\prime} \in\left[T_{k-1}, T_{k}\right]} \int\left(u_{k}^{+}\left(t^{\prime}\right)\right)^{2} d x & \leq \frac{1}{T_{k}-T_{k-1}} \int_{T_{k-1}}^{T_{k}} \int\left(u_{k}^{+}\left(t^{\prime}\right)\right)^{2} d x d t^{\prime} \\
& \leq 2^{k} \int_{T_{k-1}}^{T_{k}} \int\left(u_{k}^{+}\left(t^{\prime}\right)\right)^{2} d x d t^{\prime}
\end{aligned}
$$

Observing that $u_{k}^{+}(x)>0$ implies $u_{k-1}^{+}(x)>u_{k}^{+}(x)+4^{-k} / 2$, we can realize that both $I$ and $I I$ have the same flavour, and that in fact we have the estimate

$$
\mathcal{A}_{k} \leq C^{k} \int_{T_{k-1}}^{0} \int_{u_{k-1}^{+}>4^{-k} / 2}\left(u_{k-1}^{+}\right)^{2} d x d t,
$$

for a possibly larger constant $C$. The next step is to modify the proof in Section 4.2, replacing the Sobolev inequality by the second part of Lemma 3.2. To that end, let $p>2$ be the exponent corresponding to Sobolev's embedding theorem so that

$$
\int\left(u_{k-1}^{+}\right)^{p} d x \leq C\left[\mathcal{B}\left(u_{k-1}^{+}, u_{k-1}^{+}\right)\right]^{p / 2} .
$$

Take $\theta=2 / p$ and define $q=(1-\theta) 2+\theta p$. Then

$$
\begin{aligned}
\int_{u_{k-1}^{+}>4^{-k} / 2}\left(u_{k-1}^{+}\right)^{2} d x & \leq 4^{(k+1)(q-2)} \int\left(u_{k-1}^{+}\right)^{q} d x \\
& \leq 4^{(k+1)(q-2)}\left(\int\left(u_{k-1}^{+}\right)^{2} d x\right)^{1-\theta}\left(\int\left(u_{k-1}^{+}\right)^{p} d x\right)^{\theta} \\
& \leq C 4^{k(q-2)}\left(\int\left(u_{k-1}^{+}\right)^{2} d x\right)^{1-\theta} \mathcal{B}\left(u_{k-1}^{+}, u_{k-1}^{+}\right)
\end{aligned}
$$

Integration in time $t$ along the interval $\left[T_{k-1}, 0\right]$ gives us from inequality (6.13), and the previous estimate a recurrence relation of the form

$$
\begin{aligned}
\mathcal{A}_{k} & \leq C^{k}\left(\sup _{T_{k-1} \leq t \leq 0} \int\left(u_{k-1}^{+}(t)\right)^{2} d x\right)^{1-\theta} \cdot \int_{T_{k-1}}^{0} \mathcal{B}\left(u_{k-1}^{+}, u_{k-1}^{+}\right) d t \\
& \leq C^{k} \mathcal{A}_{k-1}^{1-\theta} \mathcal{A}_{k-1}=C^{k} \mathcal{A}_{k-1}^{1+\tau},
\end{aligned}
$$

with $\tau=1-\theta>0$ and a possibly larger constant $C$. 
We need $\delta$ to be very small to start the iteration so that the sequence $\mathcal{A}_{k}$ converges and then $\mathcal{A}_{\infty}=0$, which means that $u \leq \varphi_{\infty}$, and this in turn implies that $u \leq 7 / 8$ for $|x| \leq 1$. We thus get the result in the statement of the lemma with $\mu=1 / 8$.

Remark. A simple modification of $\varphi_{\infty}$ would give other values of $\mu \in(0,1 / 2)$, of course with a different estimate of the maximum allowed value for $\delta$. The proof also shows that the time size $T=4$ can be replaced by any other number and the iteration will work with a different value for $\delta$ (and the same values for $\mu$ and $\varepsilon$ ).

\section{Modification of the energy calculation}

In the iterative process that we will consider below, in Section 11, there will be situations in which the solution considered in a cylinder as above is bounded between two positive constants $0<M_{1}<M_{2}$. We want to establish that a similar result holds and the $\delta$ - $\mu$ relation does not change much, which will be essential in our iterations. The use of rescaling and the translation invariance in $(x, t)$ allow one to recover a solution defined in the standard domain $\Gamma_{4}$ which is the one chosen for all our calculations. But imposing the normalization $0 \leq u \leq 1$ asks for a vertical translation in $u$ to adjust the lower level (on top of the usual scaling), and this leads to a modified equation

$$
\partial_{t} u=\nabla \cdot(D(u) \nabla \mathcal{L} u)
$$

where $D$ has the form $D(u)=d_{1}+d_{2} u$. We will normalize so that $d_{1}+d_{2}=1$ (i.e., $D(1)=1$ ) and we will have $\left|d_{2}\right|<1$ (in practice, $d_{2}$ becomes small as the iterations advance).

We re-do the energy calculations of the previous section but this time we use as test function $\eta=F(u \vee \varphi)-F(\varphi)$, where $F$ is defined as

$$
F(u):=\int_{1}^{u} \frac{1}{D(s)} d s=\frac{1}{d_{2}} \log \left(d_{1}+d_{2} u\right) .
$$

Note that $\eta \geq 0$ and $\eta=0$ where $u \leq \varphi$. Then the LHS gives

$$
I_{\mathrm{lhs}}=\int_{T_{1}}^{T_{2}} \int \eta \partial_{t} u d x d t=\frac{1}{d_{2}} \int_{T_{1}}^{T_{2}} \int \log \left(\frac{d_{1}+d_{2}(u \vee \varphi)}{d_{1}+d_{2} \varphi}\right) \partial_{t} u d x d t .
$$

Next, we write

$$
\frac{d_{1}+d_{2}(u \vee \varphi)}{d_{1}+d_{2} \varphi}=1+\beta u_{\varphi}^{+}, \quad \beta=\frac{d_{2}}{d_{1}+d_{2} \varphi},
$$

and note that the function

$$
H(s):=\frac{1}{d_{2}}\left\{s \log (1+\beta s)+\frac{1}{\beta} \log (1+\beta s)-s\right\}
$$

is such that $H^{\prime}(s)=d_{2}^{-1} \log (1+\beta s)$, so that by integration in time we arrive at

$$
I_{\mathrm{lhs}}=\int H\left(u_{\varphi}^{+}\left(x, T_{2}\right)\right) d x-\int H\left(u_{\varphi}^{+}\left(x, T_{1}\right)\right) d x .
$$


We will need an estimate of this quantity. Since $H(0)=H^{\prime}(0)=0$ and $H^{\prime \prime}(s)=$ $\beta /\left(d_{2}(1+\beta s)\right)$, i.e., $H^{\prime \prime}\left(u_{\varphi}^{+}\right)=1 /\left(d_{1}+d_{2}(u \vee \varphi)\right)$, we have the estimate $H\left(u_{\varphi}^{+}\right) \sim\left(u_{\varphi}^{+}\right)^{2}$ at all points where $u \geq \varphi, u$ is bounded above and $\varphi$ is bounded below away from zero in the sense that

$$
c_{1}\left(u_{\varphi}^{+}\right)^{2} \leq H\left(u_{\varphi}^{+}\right) \leq c_{2}\left(u_{\varphi}^{+}\right)^{2}
$$

and the constants go to $1 / 2$ as $d_{2} \rightarrow 0$ (and $d_{1} \rightarrow 1$ ), since in the limit $H^{\prime \prime}(s)=1$.

On the other hand, on the RHS of the weak formulation, instead of (6.4), we have

$$
\begin{aligned}
\int d t & \int(F(u \vee \varphi)-F(\varphi)) \operatorname{div}[D(u(x)) \nabla L(x-y)(u(x)-u(y))] d x d y \\
& =-\int d t \int_{u>\varphi}\left\{\frac{\nabla u(x)}{D(u(x))}-\frac{\nabla \varphi(x)}{D(\varphi(x))}\right\} D(u(x)) \nabla_{x} L(x-y)(u(x)-u(y)) d x d y,
\end{aligned}
$$

which we again split as I + II. In the present situation we take

$$
\mathbf{I}=-\int d t \iint \nabla u_{\varphi}^{+}(x) \nabla_{x} L(x-y)[u(y)-u(x)] d x d y .
$$

After integrating by parts and symmetrizing, we get $-\mathbf{I}=\frac{1}{2} \int \mathcal{B}\left(u_{\varphi}^{+}, u\right) d t$, where

$$
\mathcal{B}\left(u_{\varphi}^{+}, u\right)=\iint\left(u_{\varphi}^{+}(x)-u_{\varphi}^{+}(y)\right) K(x-y)(u(x)-u(y)) d x d y .
$$

As before, we separate the expression into three integrals, using the splitting $u=u_{\varphi}^{+}+$ $\varphi+u_{\varphi}^{-}$. The rest of the integral on the RHS takes the form

$$
\mathbf{I I}=\int d t \iint_{u(x)>\varphi(x)} \frac{D(u)-D(\varphi)}{D(\varphi)} \nabla \varphi(x) \nabla L(x-y)(u(x)-u(y)) d x d y .
$$

- When $D(u):=d_{1}+d_{2} u$ with $d_{1}+d_{2}=1$, we have $F(s)=\left(1 / d_{2}\right) \log \left(d_{1}+d_{2} u\right)$ and $\mathbf{I I}=\int \widehat{\mathcal{Q}}\left(u_{\varphi}^{+}, u\right) d t$ with

$$
\widehat{\mathcal{Q}}\left(u_{\varphi}^{+}, u\right)=d_{2} \iint u_{\varphi}^{+}(x) \frac{\nabla \varphi(x)}{D(\varphi(x))} \nabla L(x-y)(u(x)-u(y)) d x d y,
$$

which looks like $\mathcal{Q}$ of the previous section but for an interesting small factor, $d_{2}$.

Finally, the energy inequality takes the form

$$
\begin{aligned}
\left.\int H\left(u_{\varphi}^{+}\right) d x\right|_{T_{1}} ^{T_{2}}+C \int_{T_{1}}^{T_{2}} \mathcal{B}\left(u_{\varphi}^{+}, u_{\varphi}^{+}\right) d t+C \int_{T_{1}}^{T_{2}} \mathcal{B}\left(u_{\varphi}^{+}, u_{\varphi}^{-}\right) d t \\
\leq C_{2} \int_{T_{1}}^{T_{2}} \mathcal{B}\left(u_{\varphi}^{+}, \varphi\right) d t+\int_{T_{1}}^{T_{2}} \widehat{\mathcal{Q}}\left(u_{\varphi}^{+}, u\right) d t
\end{aligned}
$$

to be compared with identity (6.5).

Repeating the rest of the steps of the previous section offers no novelties and we arrive at a similar result: 
Lemma 7.1. The result of Lemma 5.1 holds for the weak energy solutions of (7.1) under the assumptions $D(u)=d_{1}+d_{2} u, d_{1}, d_{2} \geq 0, d_{1}+d_{2}=1, d_{1} \geq 1 / 2$, and the result holds with the same constants $\mu, \delta$ for different values of $d_{1}, d_{2}$.

\section{Pulling up from zero. Proof of Lemma 5.2}

Here, we are trying to pull the equation uniformly away from zero under some assumption on the size of a level set. The precise assumptions are

$$
0 \leq u \leq \max \left\{1,|x|^{\varepsilon}-1\right\} \quad \text { in } \mathbb{R}^{N} \times[-4,0],
$$

and $\left|\{u \leq 1 / 2\} \cap \Gamma_{4}\right| \leq \delta\left|\Gamma_{4}\right|$. The desired conclusion is then that $\left.u\right|_{\Gamma_{1}} \geq \mu_{0}>0$, for an appropriate $\delta>0$ to be chosen.

The tools are energy inequalities and integration by parts. In order to deduce the necessary energy inequalities we recall the bilinear forms and integration by parts formulas of Section 6. We use the same notation for the kernels $K$ and $L$.

\subsection{Local energy inequality}

The basic calculation is as follows. We take a positive smooth function $\varphi$ and use $\eta=$ $\log v$ as a test function in the weak formulation, where $v(x, t)$ is defined as $v=u / \varphi$ if $u<\varphi$ and $v=1$ otherwise. In other words,

$$
v=\frac{u}{\varphi} \wedge 1, \quad \text { or } \quad v-1=\frac{(u-\varphi)^{-}}{\varphi}=: \frac{u_{\varphi}^{-}}{\varphi} .
$$

Note that $0 \leq v \leq 1$. We will use the notations $u_{\varphi}^{+}=(u-\varphi)^{+}, u_{\varphi}^{-}=(u-\varphi)^{-}$, so that $u=\varphi+u_{\varphi}^{+}+u_{\varphi}^{-}$. Next, we put

$$
h(v)=v \log v-(v-1), \quad \forall v \geq 0 .
$$

This function takes as minimum $h(1)=0$ and $h(v)>0$ for all $v>0, v \neq 1$ with $h(0)=1$. Moreover, $h^{\prime \prime}(v)=1 / v$ so that for $0<v \leq 1$ we have $h^{\prime \prime}(v) \geq 1$ and

$$
h(v)=v \log v+1-v \geq \frac{1}{2}(v-1)^{2}=\frac{\left(u_{\varphi}^{-}\right)^{2}}{2 \varphi^{2}} .
$$

We also have the inequality

$$
h(v) \leq(v-1)^{2}=\frac{\left(u_{\varphi}^{-}\right)^{2}}{\varphi^{2}} .
$$

This is used below. Next, we have the following calculation for the choice of $v=v(x, t)$ made in (8.1):

$$
\frac{d}{d t} \int \varphi h(v) d x=\int \log (v) \varphi v_{t}=\int_{u<\varphi} \log (v) \varphi v_{t}=\int \log (v) u_{t},
$$


since $\log (v)=0$ for $u \geq \varphi$. Using the weak energy formulation of the equation, we now get

$$
\int \varphi h\left(v\left(t_{2}\right)\right) d x+\int_{t_{1}}^{t_{2}} \int \nabla[\log (v)] u \nabla \mathcal{L} u d x d t=\int \varphi h\left(v\left(t_{1}\right)\right) d x .
$$

Let us work out the meaning of the middle term (energy term). First, we have

$$
\begin{aligned}
\int \nabla[\log (v)] \cdot u \nabla \mathcal{L} u d x=\int & u \frac{\nabla v}{v} \cdot \nabla \mathcal{L} u d x=\int_{u<\varphi} \varphi \nabla\left(u_{\varphi}^{-} / \varphi\right) \cdot \nabla \mathcal{L} u d x \\
= & \int_{u<\varphi} \nabla u_{\varphi}^{-} \cdot \nabla \mathcal{L} u d x-\int_{u<\varphi} \frac{u_{\varphi}^{-}}{\varphi} \nabla \varphi \cdot \nabla \mathcal{L} u d x .
\end{aligned}
$$

Clearly, using $u=\varphi+u_{\varphi}^{+}+u_{\varphi}^{-}=(u \vee \varphi)+u_{\varphi}^{-}$, we get

$$
\int_{u<\varphi} \nabla u_{\varphi}^{-} \cdot \nabla \mathcal{L} u d x=\mathcal{B}\left(u_{\varphi}^{-}, u\right)=\mathcal{B}\left(u_{\varphi}^{-}, u_{\varphi}^{-}\right)+\mathcal{B}\left(u_{\varphi}^{-}, u_{\varphi}^{+}\right)+\mathcal{B}\left(u_{\varphi}^{-}, \varphi\right) .
$$

The first term is the one we want to keep to complete the expression of the energy. The second term $\mathcal{B}\left(u_{\varphi}^{-}, u_{\varphi}^{+}\right)$is also positive in view of (3.1) since the two functions have opposite signs and disjoint supports (cf. Corollary 3.1). Hence, this term could be discarded, but it will turn out to be useful as we have already seen.

The remaining term $\mathcal{B}\left(u_{\varphi}^{-}, \varphi\right)$ and the last integral in (8.5) are delicate and it is there that we have to make an argument with a careful choice of test function. Summing up, we have

$\int \nabla[\log (v)] \cdot u \nabla \mathcal{L} u d x \geq \mathcal{B}\left(u_{\varphi}^{-}, u_{\varphi}^{-}\right)+\mathcal{B}\left(u_{\varphi}^{-}, \varphi\right)+\mathcal{B}\left(u_{\varphi}^{-}, u_{\varphi}^{+}\right)-\int_{u<\varphi} \frac{u_{\varphi}^{-}}{\varphi} \nabla \varphi \cdot \nabla \mathcal{L} u d x$.

Putting

$$
\mathcal{Q}(w, u)=\iint w(x) \frac{\nabla \varphi(x)}{\varphi(x)} \nabla L(x-y)[u(y)-u(x)] d x d y,
$$

from the above and from (8.2) and (8.3) we arrive of the basic energy inequality:

$$
\begin{array}{rl}
\int \frac{1}{2 \varphi}\left(u_{\varphi}^{-}\left(t_{2}\right)\right)^{2} & d x+\int_{t_{1}}^{t_{2}} \mathcal{B}\left(u_{\varphi}^{-}, u_{\varphi}^{-}\right) d t+\int_{t_{1}}^{t_{2}} \mathcal{B}\left(u_{\varphi}^{-}, u_{\varphi}\right) d t \\
\leq & \int \frac{1}{\varphi}\left(u_{\varphi}^{-}\left(t_{1}\right)\right)^{2} d x-\int_{t_{1}}^{t_{2}} \mathcal{B}\left(u_{\varphi}^{-}, \varphi\right) d t+\int_{t_{1}}^{t_{2}} \mathcal{Q}\left(u_{\varphi}^{-}, u\right) d t .
\end{array}
$$

The local energy, in the time interval $\left[T_{1}, T_{2}\right]$, is now defined as

$$
E_{\varphi}(u):=\sup _{T_{1}<t<T_{2}} \int \frac{1}{\varphi}\left[u_{\varphi}^{-}\right]^{2} d x+\int_{T_{1}}^{T_{2}} \mathcal{B}\left(u_{\varphi}^{-}, u_{\varphi}^{-}\right) d t .
$$

Note that the test function $\log v$ is negative but we are interested in $u$ being a supersolution, and this is the case for instance if we truncate it by $2: \bar{u}=u \wedge 2$. So, we do not need to worry about growth at infinity. 


\subsection{The iterative process}

(i) At this point, following De Giorgi's idea we want to obtain an iterative relation playing the energy inequality against the Sobolev embedding using a convenient sequence of cutoff functions. We consider a series of cutoffs with dyadic separation, so that

$$
\varphi_{k+1} \leq \varphi_{k}-a 2^{-k}
$$

in the support of $\varphi_{k+1}$ for some fixed $0<a<1$. Moreover, the $\varphi_{k}$ converge to $\mu_{0}$ from above in $B_{2}(0)$. We will use as a test function $\eta=\log v$ with $v=u / \varphi_{k}$ if $u<\varphi_{k}$ and $v=1$ otherwise. We arrive at the energy inequality at the end of the last subsection with $\varphi_{k}$ instead of $\varphi$.

(ii) We propose a concrete construction of the cutoffs $\varphi_{k}$. All functions $\varphi_{k}(x)$ are symmetric, nonnegative, nonincreasing and compactly supported and the sequence is nonincreasing in $k$. The basic profile $\varphi_{0}$ is a kind of rounded mesa:

$$
\varphi_{0}(|x|) \begin{cases}=\frac{1}{2}[4-|x|]^{m} & \text { when } 7 / 2<|x|<4, \\ \equiv 1 & \text { for }|x| \leq 3, \\ \text { a } C^{2} \text { decreasing radial function for } 3 \leq|x|<4,\end{cases}
$$

for some $m \geq 2$ to be chosen later.

To construct $\varphi_{k}$ we first rescale the graph of $\varphi_{0}$ from the interval $[3,4]=[2+1,2+2]$ to the interval $\left[2+2^{-k}, 2+2^{-k+1}\right]$, and extend by $\mu_{0}+\left(1-\mu_{0}\right) 2^{-k}$ inside the ball $B_{2+2^{-k}}$. Then $\varphi_{k}$ has the following properties:

(a) $\varphi_{k}(x) \leq \varphi_{k-1}(x) \leq \cdots \leq \varphi_{0}(x)$,

(b) $\left|\nabla \varphi_{k}\right| / \varphi_{k} \leq C^{k} \varphi_{k}^{-1 / m}$,

(c) $\varphi_{k-1}-\varphi_{k} \geq\left(1-\mu_{0}\right) 2^{-k}$ in the support of $\varphi_{k}$,

(d) $\varphi_{k} \rightarrow \mu_{0} \chi_{B_{2}}$ as $k \rightarrow \infty$,

so that $a=1-\mu_{0}$.

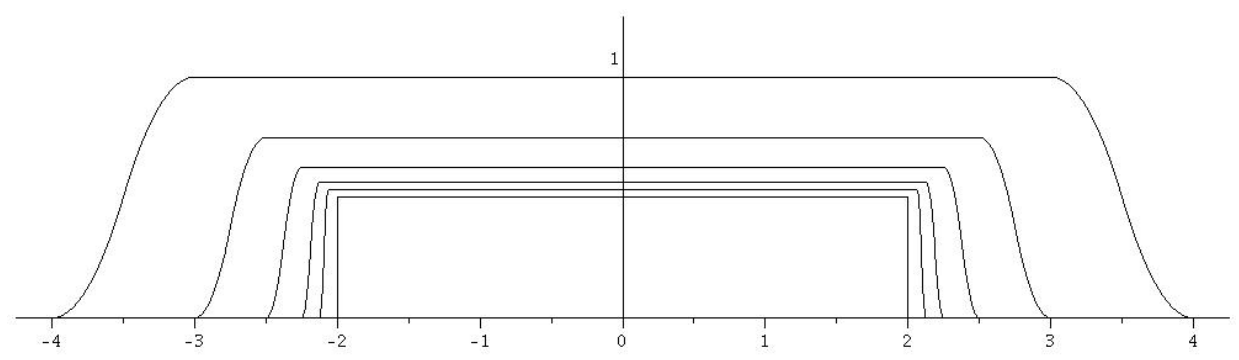

An explicit expression for $\varphi_{k}$ could be the following:

$$
\varphi_{k}(x)=\left(\mu_{0}+\left(1-\mu_{0}\right) 2^{-k}\right) \cdot \varphi_{0}\left(2^{k}|x|-2^{k+1}+2\right) .
$$

(iii) Sobolev embedding. The embedding that we need is a variant of the one used in preceding sections. We have seen that $\mathcal{B}\left(u_{\varphi}^{-}, u_{\varphi}^{-}\right)$is the square $H^{r}$ norm of $u_{\varphi}^{-}$, and in this 
way it controls the $L^{p}$ norm of $u_{\varphi}^{-}$for a given $p>1$ that we have calculated. Coupling this with the energy term

$$
\sup _{T_{1}<t<T_{2}} \int \frac{1}{\varphi}\left[u_{\varphi}^{-}\right]^{2} d x
$$

we can control, for some $q>2$ and $\theta>0$,

$$
\left(\int_{T_{1}}^{T_{2}} \int \varphi^{-\theta}\left|u_{\varphi}^{-}\right|^{q} d x d t\right)^{2 / q}
$$

(Since the cutoff $\varphi$ goes to zero, the term $\varphi^{-\theta}$ will be important in controlling the term containing $\nabla \varphi / \varphi$.) The proof is as follows: Write $q$ as a convex combination, $q=2 \theta+$ $p(1-\theta)$. Then

$$
\int \varphi^{-\theta}\left|u_{\varphi}^{-}\right|^{q} d x \leq\left(\int \varphi^{-1}\left|u_{\varphi}^{-}\right|^{2} d x\right)^{\theta}\left(\int\left|u_{\varphi}^{-}\right|^{p} d x\right)^{1-\theta} .
$$

We make the choice $\theta=(p-2) / p$ with $p$ as the $q$ of Lemma 3.2. Then $1+\theta=q / 2$ and

$$
\left(\int_{T_{1}}^{T_{2}} \int \varphi^{-\theta}\left|u_{\varphi}^{-}\right|^{q} d x d t\right)^{2 / q} \leq E_{\varphi}
$$

From now on we will write $u_{k}^{+/-}$and $E_{k}$ to denote $u_{\varphi_{k}}^{+/-}$and $E_{\varphi_{k}}$ respectively.

\subsection{Iterated energies}

On the left of (8.6) we truncate in time along an increasing sequence $T_{k} \rightarrow-2$ and get, for every $T_{k-1}<t_{1}<T_{k}<t_{2}<0$,

$$
\begin{aligned}
\int \frac{1}{2 \varphi_{k}}\left(u_{k}^{-}\left(t_{2}\right)\right)^{2} d x+\int_{t_{1}}^{t_{2}} \mathcal{B}\left(u_{k}^{-}, u_{k}^{-}\right) d t+\int_{t_{1}}^{t_{2}} \mathcal{B}\left(u_{k}^{-}, u_{k}^{+}\right) d t \\
\leq \int \frac{1}{\varphi_{k}}\left(u_{k}^{-}\left(t_{1}\right)\right)^{2} d x-\int_{t_{1}}^{t_{2}} \mathcal{B}\left(u_{k}^{-}, \varphi_{k}\right) d t-\int_{t_{1}}^{t_{2}} \mathcal{Q}\left(u_{k}^{-}, u\right) d t .
\end{aligned}
$$

\subsection{Analysis of the RHS}

We now examine the terms left on the RHS of the energy inequality (8.8) that we will call $I, I I$, and III for convenience of reference. Our purpose is to show that these extra terms are either bounded by a small multiple of the LHS, so that they will be absorbed by the LHS, or they are controlled by a term of the form

$$
C^{k} \int_{T_{1}}^{T_{2}} \int \varphi^{-\theta}\left|u_{\varphi}^{-}\right|^{2} d x d t
$$


Estimating $I$. As initial times $T_{k}$ we will choose $T_{k}=-\left(2+2^{-k}\right)$, and $t_{1} \in\left[T_{k-1}, T_{k}\right]$ is the point where the value of

$$
\inf _{T_{k-1} \leq t \leq T_{k}} \int \frac{1}{\varphi_{k}}\left(u_{k}^{-}(t)\right)^{2} d x
$$

is attained. In this way we have

$$
I \leq 2^{k} \int_{-2-2^{1-k}}^{-2-2^{-k}} \int \frac{\left[\left(u-\varphi_{k}\right)^{-}\right]^{2}}{\varphi_{k}} d x d t .
$$

Since $\left|\left(u-\varphi_{k}\right)^{-} / \varphi_{k}\right|<1$ we have $\left[\left(u-\varphi_{k}\right)^{-}\right]^{2} / \varphi_{k} \leq\left|\left(u-\varphi_{k}\right)^{-}\right|$, and we can bound $I$ by

$$
C^{k} \int_{T_{k-1}}^{0} \int \chi_{\left\{u_{k}^{-}<0\right\}}
$$

Estimate of II. For this term we have

$$
\left|\mathcal{B}\left(u_{k}^{-}, \varphi_{k}\right)\right| \leq \frac{1}{2}\left[\mathcal{B}\left(u_{k}^{-}, u_{k}^{-}\right)+\mathcal{B}^{*}\left(\varphi_{k}, \varphi_{k}\right)\right]
$$

where the star indicates that we restrict the domain of integration defining $\mathcal{B}\left(\varphi_{k}, \varphi_{k}\right)$ to the points where $u_{k}^{-}(x) \neq 0$ or $u_{k}^{-}(y) \neq 0$. So this last term can be replaced by the better expression

$$
\frac{1}{2} \iint\left[\varphi_{k}(x)-\varphi_{k}(y)\right]^{2} K(x, y) \chi(x, y) d x d y,
$$

where $\chi$ is the characteristic function of the set of points $(x, y)$ where either $x \in \operatorname{supp} u_{k}^{-}$ or $y \in \operatorname{supp} u_{k}^{-}$. From the Lipschitz character of $\varphi_{k}$ and from $\left|\nabla \varphi_{k}\right| \leq C 2^{k}$ we have, for fixed $x$,

$$
\int\left[\varphi_{k}(x)-\varphi_{k}(y)\right]^{2} K(x, y) d y \leq C^{k} .
$$

Therefore, the above term may be bounded by

$$
C^{k} \int \chi_{\left\{u_{k}^{-}<0\right\}} d x \text {. }
$$

Hence,

$$
I I \leq \frac{1}{2} \int_{t_{1}}^{t_{2}} \mathcal{B}\left(u_{k}^{-}, u_{k}^{-}\right) d t+C^{k} \int_{t_{1}}^{t_{2}} \int \chi_{\left\{u_{k}^{-}<0\right\}} d x d t .
$$

The first term is absorbed by the local energy in the LHS.

Estimate of III. The remaining part of the proof is devoted to the estimate of the term

$$
\mathcal{Q}\left(u_{k}^{-}, u\right)=\iint u_{k}^{-}(x) \frac{\nabla \varphi_{k}(x)}{\varphi_{k}(x)} \cdot \nabla L(x-y)(u(y)-u(x)) d x d y
$$

Write, by the usual splitting of $u$,

$$
\mathcal{Q}\left(u_{k}^{-}, u\right)=\mathcal{Q}\left(u_{k}^{-}, u_{k}^{-}\right)+\mathcal{Q}\left(u_{k}^{-}, u_{k}^{+}\right)+\mathcal{Q}\left(u_{k}^{-}, \varphi_{k}\right)=\mathcal{Q}_{1}+\mathcal{Q}_{2}+\mathcal{Q}_{3} .
$$


For $\mathcal{Q}_{1}$, noting that

$$
|\nabla L(x, y)| \leq|x-y| K(x, y)=O\left(|x-y|^{-(N+1-2 s)}\right),
$$

we have from Hölder's inequality

$$
\begin{aligned}
\left|\mathcal{Q}_{1}\right| & =\left|\mathcal{Q}\left(u_{k}^{-}, u_{k}^{-}\right)\right|=\left|\iint u_{k}^{-}(x) \frac{\nabla \varphi_{k}(x)}{\varphi_{k}(x)} \nabla L(x-y)\left[u_{k}^{-}(y)-u_{k}^{-}(x)\right] d x d y\right| \\
& \leq\left[\iint\left|u_{k}^{-}(x) \frac{\nabla \varphi_{k}(x)}{\varphi_{k}(x)}\right|^{2} \frac{1}{|x-y|^{N-2 s}} d x d y\right]^{1 / 2} \cdot\left[\mathcal{B}\left(u_{k}^{-}, u_{k}^{-}\right)\right]^{1 / 2} \\
& \leq 4 C_{N} \int \varphi_{k}^{-2 / m}\left(u_{k}^{-}\right)^{2} d x+\frac{1}{4} \mathcal{B}\left(u_{k}^{-}, u_{k}^{-}\right),
\end{aligned}
$$

where we have used the properties of the functions $\varphi_{k}$. Integration in time gives that the second term is absorbed by the local energy expression on the LHS, and the first is admissible for our purposes.

Final step. To study $\mathcal{Q}_{2}$ and $\mathcal{Q}_{3}$ we consider a smooth decomposition of the kernel

$$
\nabla L=\nabla L_{\psi}+\nabla L_{1-\psi}
$$

where $1-\psi$ is a bump function supported around the origin. We get

$$
\begin{aligned}
\mathcal{Q}_{2}+\mathcal{Q}_{3}= & \iint u_{k}^{-}(x) \frac{\nabla \varphi_{k}(x)}{\varphi_{k}(x)} \nabla L_{1-\psi}(x-y)\left[u_{k}^{+}(y)+\varphi_{k}(y)\right] d x d y \\
& +\iint u_{k}^{-}(x) \frac{\nabla \varphi_{k}(x)}{\varphi_{k}(x)} \nabla L_{\psi}(x-y)[u(y)] d x d y=: \overline{\mathcal{Q}}_{2}+\overline{\mathcal{Q}}_{3} .
\end{aligned}
$$

In other words, in the compact part of the support of $1-\psi$ we keep the expression of $u$ as $u_{k}^{-}+\varphi_{k}+u_{k}^{+}$, while outside, where $u_{k}^{-}=0$ and $\varphi_{k}$ grows, we just keep the term

$$
\int \nabla L_{\psi}(x-y) u(y) d y \text {. }
$$

- This term has to be controlled in a different way (through the change of coordinates if $s \geq 1 / 2$ ). As a consequence, the integrations in $y$ for $\overline{\mathcal{Q}}_{2}$ and $\overline{\mathcal{Q}}_{3}$ are convergent and we are left with estimating

$$
\int\left|u_{k}^{-}(x) \frac{\nabla \varphi_{k}(x)}{\varphi_{k}(x)}\right| d x \leq \int \varphi_{k}^{-2 / m}\left(u_{k}^{-}\right)^{2} d x+\int \chi_{\left\{u_{k}^{-}<0\right\}} d x .
$$

The integration in time produces again two admissible terms.

- Finally, for the term

$$
\iint u_{k}^{-}(x) \frac{\nabla \varphi_{k}(x)}{\varphi_{k}(x)} \nabla L_{1-\psi}(x-y)\left[u_{k}^{+}(y)\right] d x d y,
$$


we use the good term

$$
\mathcal{B}\left(u_{k}^{-}, u_{k}^{+}\right)=\iint u_{k}^{-}(x) K(x-y)\left[u_{k}^{+}(y)\right] d x d y,
$$

left in the energy inequality, to absorb the integral whenever

$$
\left|\frac{\nabla \varphi_{k}(x)}{\varphi_{k}(x)}\right||x-y| \leq \eta
$$

In the complement, that is, when

$$
\left|\frac{\nabla \varphi_{k}(x)}{\varphi_{k}(x)}\right||x-y| \geq \eta
$$

we use $\varphi_{k}^{-1 / m} \geq\left|\nabla \varphi_{k}\right| / \varphi_{k}$ and integrate in $y$ :

$$
\left|\int \nabla L_{1-\psi}(x-y)\left[u_{k}^{+}(y)\right] d y\right| \lesssim \int_{\eta \varphi_{k}^{1 / m}}^{4} \frac{r^{n-1}}{r^{n+s-1}} d r \sim \max \left(C, \eta \varphi_{k}^{(1-s) / m}\right) .
$$

In any case, we end up in the worst of the cases with an expression of the form

$$
\int \varphi_{k}^{-s / m}\left|u_{k}^{-}\right| d x
$$

which we control as before.

Inserting estimates $I I$ and $I I I$ in (8.8) we get

$$
\begin{aligned}
& \int \frac{1}{2 \varphi_{k}}\left(u_{k}^{-}\left(t_{2}\right)\right)^{2} d x+\int_{t_{1}}^{t_{2}} \mathcal{B}\left(u_{k}^{-}, u_{k}^{-}\right) d t \\
& \leq \int \frac{1}{\varphi_{k}}\left(u_{k}^{-}\left(t_{1}\right)\right)^{2} d x+C^{k}\left[\int_{t_{1}}^{t_{2}} \int \varphi_{k}^{-2 / m}\left(u_{k}^{-}\right)^{2} d x d t+\int_{t_{1}}^{t_{2}} \int \chi_{\left\{u_{k}^{-}<0\right\}} d x d t\right]
\end{aligned}
$$

whenever $T_{k-1}<t_{1}<T_{k}<t_{2}<0$. In particular,

$$
\begin{aligned}
& \sup _{T_{k}<t_{2}<0} \int \frac{1}{\varphi_{k}}\left(u_{k}^{-}\left(t_{2}\right)\right)^{2} d x+\int_{T_{k}}^{0} \mathcal{B}\left(u_{k}^{-}, u_{k}^{-}\right) d t \leq \inf _{T_{k-1}<t_{1}<T_{k}} \int \frac{2}{\varphi_{k}}\left(u_{k}^{-}\left(t_{1}\right)\right)^{2} d x \\
&+ C^{k}\left[\int_{T_{k-1}}^{0} \int \varphi_{k}^{-2 / m}\left(u_{k}^{-}\right)^{2} d x d t+\int_{T_{k-1}}^{0} \int \chi_{\left\{u_{k}^{-}<0\right\}} d x d t\right] \\
& \leq C^{k}\left[\int_{T_{k-1}}^{0} \int \varphi_{k}^{-2 / m}\left(u_{k}^{-}\right)^{2} d x d t+\int_{T_{k-1}}^{0} \int \chi_{\left\{u_{k}^{-}<0\right\}} d x d t\right],
\end{aligned}
$$


where the last inequality comes from estimate $I$. In fact, the same argument given for this estimate shows that if $m \geq 2$ then the leading term in the last expression is the second one. Thus, if we define

$$
\mathcal{A}_{k}:=\sup _{T_{k}<t<0} \int\left(u_{k}^{-}(t)\right)^{2} d x+\int_{T_{k}}^{0} \mathcal{B}\left(u_{k}^{-}, u_{k}^{-}\right) d t
$$

then (8.10) gives

$$
\mathcal{A}_{k} \leq C^{k} \int_{T_{k-1}}^{0} \int \chi_{\left\{u_{k}^{-}<0\right\}} d x d t .
$$

Using that $\varphi_{k-1} \geq \varphi_{k}+a 2^{-k}$ in the support of $\varphi_{k}$ we then get $\left|u_{k-1}^{-}\right| \geq\left|u_{k}^{-}\right|+a 2^{-k}$ in $\left\{u_{k}^{-}<0\right\}$. Therefore,

$$
\mathcal{A}_{k} \leq C^{(1+q) k} \int_{T_{k-1}}^{0} \int\left|u_{k-1}^{-}\right|^{q} d x d t
$$

The rest follows the same argument as in Lemma 5.1: Let $p>2$ be the exponent corresponding to Sobolev's embedding theorem so that

$$
\int\left|u_{k-1}^{-}\right|^{p} d x \leq C\left[\mathcal{B}\left(u_{k-1}^{-}, u_{k-1}^{-}\right)\right]^{p / 2}
$$

Take $\theta=(p-2) / p$ and define $q=\theta 2+(1-\theta) p$. Then

$$
\begin{aligned}
\mathcal{A}_{k} & \leq C^{(1+q) k} \int_{T_{k-1}}^{0}\left(\int\left|u_{k-1}^{-}\right|^{2} d x\right)^{\theta} \mathcal{B}\left(u_{k-1}^{-}, u_{k-1}^{-}\right) d t \\
& \leq \widetilde{C}^{k}\left(\sup _{T_{k-1} \leq t \leq 0} \int\left(u_{k-1}^{-}(t)\right)^{2} d x\right)^{\theta} \cdot \int_{T_{k-1}}^{0} \mathcal{B}\left(u_{k-1}^{-}, u_{k-1}^{-}\right) d t \\
& \leq \widetilde{C}^{k} \mathcal{A}_{k-1}^{\theta} \mathcal{A}_{k-1}=\widetilde{C}^{k} \mathcal{A}_{k-1}^{1+\theta} .
\end{aligned}
$$

This completes the proof of Lemma 5.2 for the critical case since, by hypothesis,

$$
A_{0}=\int_{-4}^{0} \int \frac{\left[\left(u-\varphi_{0}\right)^{-}\right]^{2}}{\varphi_{0}} d x d t \leq \iint_{\Gamma_{4}} \chi_{\left\{u-\varphi_{0}<0\right\}} \leq\left|\{u \leq 1\} \cap \Gamma_{4}\right| \leq \delta\left|\Gamma_{4}\right|,
$$

and $\delta$ can be chosen as small as we need.

The noncritical case of Lemma 5.2. This is the case where the solution lies between, say, $M$ and $M+1$ for some $M>0$. The proof is similar except that for the lower estimate we may already assume that $\left.u\right|_{\Gamma_{4}} \geq \mu>0$ and then all the cutoffs involved satisfy $\varphi_{k}>\mu$ so that $\nabla \varphi_{k} / \varphi_{k}$ is a smooth bounded function. 


\section{The lemma on intermediate values}

We still need a main ingredient before we attack the regularity issue by means of a suitable iteration. Indeed, we have to improve Lemma 5.1 by showing that, in order to get a uniform reduction of the maximum in a smaller ball it is not necessary to ask that $u \leq 1 / 2$ "most of the time" in $\Gamma_{4}$, but only "some of the time". This is precisely stated in Lemma 5.3.

The proof of this result uses De Giorgi's idea of loss of mass at intermediate levels, which he applied in an elliptic context. In the present parabolic setting we will follow the idea of the proof of the similar result that was carried out in the papers [10], [6] in the linear framework. We next give the detailed statement of the "intermediate lemma" and its proof. We start by selecting a cutoff function of the form $\varphi=1+\psi_{\lambda}+F$, where we choose

$$
F(x)=\sup \left\{-1, \inf \left\{0,|x|^{2}-9\right\}\right\} .
$$

Note that $F \leq 0$ is Lipschitz, compactly supported in $B_{3}(0)$ and $F=-1$ in $B_{2}(0)$. Moreover, for $0<\lambda<1 / 3$ we define

$$
\psi_{\lambda}(x)=\left(\left(|x|-\lambda^{-1 / v}\right)^{v}-1\right)^{+} \quad \text { for }|x| \geq \lambda^{-1 / v},
$$

and $\psi_{\lambda}(x)=0$ otherwise. This represents a "wing" that starts far away when $\lambda$ is small, as will be the case. A convenient value of $v \in(0,1)$ is needed and it will be determined later. We also define

$$
\varphi_{1}=1+\psi_{\lambda}+\lambda F, \quad \varphi_{2}=1+\psi_{\lambda}+\lambda^{2} F
$$

and put $\varphi_{0}=\varphi$, so that $\varphi_{0} \leq \varphi_{1} \leq \varphi_{2} \leq 1$ in the ball $B_{4}(0)$.

Lemma 9.1. There exist small constants $\rho, \gamma>0$, depending only on $N$ and $s$, and $\lambda_{0} \in(0,1)$, depending only on $N, s, \rho, \delta$, such that for any solution u defined in $S_{4}$ with

$$
u(x, t) \leq 1+\psi_{\lambda} \quad \text { in } S_{4}
$$

with $\lambda \leq \lambda_{0}$, and also such that $\left|\left\{u<\varphi_{0}\right\} \cap\left(B_{1} \times(-4,-2)\right)\right| \geq \rho$, we have the following implication: if

$$
\left|\left\{u>\varphi_{2}\right\} \cap\left(\mathbb{R}^{N} \times(-2,0)\right)\right| \geq \delta,
$$

then

$$
\left|\left\{\varphi_{0} \leq u \leq \varphi_{2}\right\} \cap\left(\mathbb{R}^{N} \times(-3,0)\right)\right| \geq \gamma
$$

The last line asserts that under the stated assumptions the measure of the intermediate level cannot be small.

Proof. (i) In our context we start from the energy estimates we have obtained during the proof of Lemma 5.1. We have to arrive at a "correct form" of the energy inequality. We 
recover the energy calculation (6.5) with $\varphi$ equal to the "intermediate cutoff" $\varphi_{1}$, i.e.,

$$
\begin{array}{r}
\left.\int \varphi_{1} H(g) d x\right|_{T_{1}} ^{T_{2}}+\frac{1}{2} \int_{T_{1}}^{T_{2}} \mathcal{B}\left(\left(u-\varphi_{1}\right)^{+},\left(u-\varphi_{1}\right)^{+}\right) d t+\frac{1}{2} \int_{T_{1}}^{T_{2}} \mathcal{B}\left(\left(u-\varphi_{1}\right)^{+},\left(u-\varphi_{1}\right)^{-}\right) d t \\
\leq-\frac{1}{2} \int_{T_{1}}^{T_{2}} \mathcal{B}\left(\left(u-\varphi_{1}\right)^{+}, \varphi_{1}\right) d t+\int_{T_{1}}^{T_{2}} \mathcal{Q} d t,
\end{array}
$$

with $g=1+\left(u-\varphi_{1}\right)^{+} / \varphi_{1}$ and $H(g)=g \log g-(g-1)$. We now want to get estimates of the RHS that show that all the terms are either absorbed by the LHS or can be estimated above by $C \lambda^{2}$, where $C$ is a fixed constant. With this we will finally arrive at the expression

$$
\begin{aligned}
\int \varphi_{1}(g \log g+1-g) d x & \left.\right|_{T_{1}} ^{T_{2}}+c \int_{T_{1}}^{T_{2}} \mathcal{B}\left(\left(u-\varphi_{1}\right)^{+},\left(u-\varphi_{1}\right)^{+}\right) d t \\
& +c \int_{T_{1}}^{T_{2}} \mathcal{B}\left(\left(u-\varphi_{1}\right)^{+},\left(u-\varphi_{1}\right)^{-}\right) d t \leq C \lambda^{2}\left(T_{2}-T_{1}\right) .
\end{aligned}
$$

This would complete the preparatory step of the lemma.

(ii) The verification that $(9.3)$ holds is as follows. For simplicity we write $\left(u-\varphi_{1}\right)^{+}=u_{1}^{+}$ and $\left(u-\varphi_{1}\right)^{-}=u_{1}^{-}$. Repeating a bit some arguments, to prove (9.3) we observe that

$$
\left|\mathcal{B}\left(u_{1}^{+}, \varphi_{1}\right)\right| \leq \gamma \mathcal{B}\left(u_{1}^{+}, u_{1}^{+}\right)+\frac{2}{\gamma} \mathcal{B}^{*}\left(\varphi_{1}, \varphi_{1}\right)
$$

for every $\gamma>0$, where

$$
\mathcal{B}^{*}\left(\varphi_{1}, \varphi_{1}\right)=\iint \chi_{B_{3}}(x) K(x-y)\left(\varphi_{1}(x)-\varphi_{1}(y)\right)^{2} d y d x
$$

For $\gamma$ small, $\gamma \mathcal{B}\left(u_{1}^{+}, u_{1}^{+}\right)$is absorbed into the LHS of (9.2). For the second term we have

$$
\mathcal{B}^{*}\left(\varphi_{1}, \varphi_{1}\right) \leq 4\left(\lambda^{2} \mathcal{B}^{*}(F, F)+\mathcal{B}^{*}\left(\psi_{\lambda}, \psi_{\lambda}\right)\right)
$$

Since the function $F$ is Lipschitz, compactly supported and $|x-y|^{2} K(x-y \mid$ is locally integrable, we have $\mathcal{B}^{*}(F, F) \leq C$. Now, for $\lambda$ small one has $\lambda^{-1 / v} \geq 4$ so that $\psi_{\lambda}(x)=0$ if $|x|<3$. Therefore, since $\psi_{\lambda}(y) \leq|y|^{\nu}$ always, we get

$$
\begin{aligned}
\mathcal{B}^{*}\left(\psi_{\lambda}, \psi_{\lambda}\right) & =\int_{B_{3}} \int_{|y|>\lambda^{-1 / v}} K(x-y)\left(\psi_{\lambda}(y)\right)^{2} d y d x \\
& \leq \int_{B_{3}} \int_{|y|>\lambda^{-1 / v}} \frac{|y|^{2 v}}{|x-y|^{N+2-2 s}} d y d x \\
& \leq C \int_{B_{3}} \int_{|y|>\lambda^{-1 / v}} \frac{|y|^{2 v}}{|y|^{N+2-2 s}} d y d x \sim \lambda^{(2-2 s-2 v) / v} .
\end{aligned}
$$


It suffices to take $(2-2 s-2 v) / v \geq 2$, that is, $v \leq(2-2 s) / 4$, in order to conclude that

$$
\mathcal{B}^{*}\left(\varphi_{1}, \varphi_{1}\right) \leq O\left(\lambda^{2}\right) .
$$

We now consider the terms involving $\mathcal{Q}$ :

$$
\mathcal{Q}\left(u_{1}^{+}, u\right)=\mathcal{Q}\left(u_{1}^{+}, u_{1}^{+}\right)+\mathcal{Q}\left(u_{1}^{+}, \varphi_{1}\right)+\mathcal{Q}\left(u_{1}^{+}, u_{1}^{-}\right) .
$$

The main ingredients are the following estimates:

- $u_{1}^{+} \leq \lambda|F|$, since $0 \leq u \leq 1+\psi_{\lambda}$; in particular,

- $u_{1}^{+} \leq \lambda \chi_{B_{3}}$,

- $|\nabla L(x-y)| \sim|x-y| K(x-y) \sim 1 /|x-y|^{N+1-2 s}$, integrable at infinity for $s<1 / 2$,

- $\left|\nabla \varphi_{1}(x)\right| / \varphi_{1}(x) \leq C$ for all $x$, with $C$ independent of $\lambda$, and

- $\left|\nabla \varphi_{1}(x)\right| / \varphi_{1}(x) \leq C \lambda$ in the support of $u_{1}^{+}$.

Hence,

$$
\begin{gathered}
\left|\mathcal{Q}\left(u_{1}^{+}, u_{1}^{+}\right)\right|=\left|\iint u_{1}^{+}(x) \frac{\nabla \varphi_{1}(x)}{\varphi_{1}(x)} \nabla L(x-y)\left(u_{1}^{+}(x)-u_{1}^{+}(y)\right) d x d y\right| \\
\leq C \eta \mathcal{B}\left(u_{1}^{+}, u_{1}^{+}\right)+C \lambda \iint_{|x-y| \geq \eta}\left[\left(u_{1}^{+}(x)\right)^{2}+u_{1}^{+}(x) u_{1}^{+}(y)\right]|\nabla L(x-y)| d x d y \\
\leq C \eta \mathcal{B}\left(u_{1}^{+}, u_{1}^{+}\right)+C \lambda \int\left(u_{1}^{+}(x)\right)^{2} d x+C \lambda\left(\int u_{1}^{+}(x) d x\right)^{2} .
\end{gathered}
$$

The first term in the last line of the formula goes to the LHS of (9.2) for $\eta$ small and the other two are just $O\left(\lambda^{3}\right)$. Also,

$$
\mathcal{Q}\left(u_{1}^{+}, \varphi_{1}\right)=\lambda \mathcal{Q}\left(u_{1}^{+}, F\right)+\mathcal{Q}\left(u_{1}^{+}, \psi_{\lambda}\right) .
$$

Since

$$
\left|\mathcal{Q}\left(u_{1}^{+}, F\right)\right| \leq C \lambda \int u_{1}^{+}(x) \int|\nabla L(x-y)(F(x)-F(y))| d y d x \leq C \lambda \int u_{1}^{+}(x) d x,
$$

the first term above is easily seen to be of order $O\left(\lambda^{3}\right)$. For the second we have

$$
\begin{aligned}
\left|\mathcal{Q}\left(u_{1}^{+}, \psi_{\lambda}\right)\right| & \leq C \lambda \int u_{1}^{+}(x) \int\left|\nabla L(x-y)\left(\psi_{\lambda}(x)-\psi_{\lambda}(y)\right)\right| d y d x \\
& \leq C \lambda \int u_{1}^{+}(x) \int_{|y|>\lambda^{-1 / v}} \frac{|y|^{\nu}}{|y|^{N+1-2 s}} d y d x,
\end{aligned}
$$

where we have used that $\psi_{\lambda}(x)=0$ in the support of $u_{1}^{+}$and $\psi_{\lambda}(y) \leq|y|^{\nu}$. From this we get

$$
\left|\mathcal{Q}\left(u_{1}^{+}, \psi_{\lambda}\right)\right| \leq C \lambda^{1+(1-2 s-v) / v} \int u_{1}^{+}(x) d x
$$


Observing that $v<1-2 s$ from the previous condition on $v$, we conclude that

$$
\mathcal{Q}\left(u_{1}^{+}, \psi_{\lambda}\right) \leq O\left(\lambda^{2}\right) .
$$

Finally,

$$
\begin{aligned}
\left|\mathcal{Q}\left(u_{1}^{+}, u_{1}^{-}\right)\right| & =\left|\iint u_{1}^{+}(x) \frac{\nabla \varphi_{1}(x)}{\varphi_{1}(x)} \nabla L(x-y)\left(u_{1}^{-}(x)-u_{1}^{-}(y)\right) d x d y\right| \\
& \leq C \eta \mathcal{B}\left(u_{1}^{+}, u_{1}^{-}\right)+\iint_{|x-y| \geq \eta} u_{1}^{+}(x)\left|\frac{\nabla \varphi_{1}(x)}{\varphi_{1}(x)}\right|\left|\nabla L(x-y) u_{1}^{-}(y)\right| d x d y .
\end{aligned}
$$

The term $C \eta \mathcal{B}\left(u_{1}^{+}, u_{1}^{-}\right)$is absorbed into the LHS of (9.2) for $\eta$ small. For the other we use the fact that $\left|u_{1}^{-}\right| \leq \varphi_{1}=1+\lambda F+\psi_{\lambda}$. Now,

$$
\begin{aligned}
& \int u_{1}^{+}(x) \int_{|y|>\lambda-1 / v}\left|\frac{\nabla \varphi_{1}(x)}{\varphi_{1}(x)}\right| \nabla L(x-y)\left(1+\psi_{\lambda}(y)\right) \mid d x d y \\
& \quad \leq C \lambda \int u_{1}^{+}(x) \int_{|y|>\lambda^{-1 / v}} \frac{1+|y|^{\nu}}{|y|^{N+1-2 s}} d y d x \leq C \lambda^{1+(1-2 s-v) / v} \int u_{1}^{+}(x) d x
\end{aligned}
$$

whereas

$$
\begin{aligned}
& \int u_{1}^{+}(x) \int_{\left\{y:|x-y| \geq \eta|y| \leq \lambda^{-1 / v}\right\}}\left|\frac{\nabla \varphi_{1}(x)}{\varphi_{1}(x)}\right||\nabla L(x-y)(1+F(y))| d x d y \\
& \leq C \lambda \int u_{1}^{+}(x) \int_{|x-y| \geq \eta} \frac{1}{|x-y|^{N+1-2 s}} d y d x \leq C \lambda \int u_{1}^{+}(x) d x .
\end{aligned}
$$

In both cases we get $O\left(\lambda^{2}\right)$ for an appropriate $v$, which proves (9.3) as desired.

(iii) Estimate (9.3) shows a property of one-sided Lipschitz continuity in time from above for the space integral of the LHS. On the other hand, we also have the inequality

$$
\int \varphi_{1} H(g)(t) d x \leq \int \varphi_{1}(g-1)^{2}(t) d x \leq \int\left(u_{1}^{+}(x)\right)^{2} d x \leq C \lambda^{2}, \quad \forall t .
$$

Inserting this into the energy inequality (9.3) we arrive at

$$
\int_{T_{1}}^{T_{2}} \mathcal{B}\left(u_{1}^{+}, u_{1}^{-}\right) d t \leq C \lambda^{2} \quad \text { for }-4<T_{1}<T_{2}<0 .
$$

We are now in a position to apply Steps 2 and 3 of the proof of Section 4 of [6] with only technical changes that we will omit, and we will thus get Lemma 9.1. Note that the quadratic (or at least superlinear) dependence on $\lambda$ in (9.3) is absolutely necessary for the proof to work. 


\section{Oscillation reduction result}

We are now ready to prove the strong oscillation reduction result, Lemma 5.3. Though the general idea of the argument follows closely Section 5 of [6], an interesting modification is needed to accomodate the nonlinearity of the equation: in doing the scaling of the solutions in each iteration we will now find solutions of a family of related equations, and we have to use the modified estimates of Section 7, which hold for that family. At some step we will have to apply the results of the previous section to such a family. This is an easy verification that we will leave to the reader.

Instead of Lemma 5.3, we will prove the following version that will be the one used later in the regularity argument. Let us fix some notation. For $\lambda$ as in the previous section, we define, for any $\varepsilon>0$,

$$
\psi_{\varepsilon, \lambda}(x)=\left(\left(|x|-1 / \lambda^{4 / s}\right)^{\varepsilon}-1\right)^{+} \quad \text { if }|x| \geq \lambda^{-4 / s},
$$

and zero otherwise.

Lemma 10.1. Given $\rho>0$ there exist $\varepsilon>0$ and $\mu_{1}$ such that for any solution of the FPME in $\mathbb{R}^{N} \times(-4,0)$ satisfying

$$
0 \leq u \leq 1+\psi_{\varepsilon, \lambda}
$$

and assuming that

$$
\left|\left\{u<\varphi_{0}\right\} \cap\left(B_{1} \times(-4,-2)\right)\right|>\rho,
$$

we have

$$
\sup _{B_{1} \times(-1,0)} u \leq 1-\mu_{1} .
$$

Note: In the next section we will take $\rho$ equal to $\delta_{0}$ as defined after the statement of Lemma 5.2.

Proof. (i) Consider $j_{0}=\left|B_{3} \times(-3,0)\right| / \gamma$, with the value of $\gamma$ given in Lemma 9.1. We fix $\varepsilon>0$ small enough so that

$$
\frac{\left(|x|^{\varepsilon}-1\right)^{+}}{\lambda^{2 j_{0}}} \leq\left(|x|^{s / 4}-1\right)^{+}
$$

for all $x$. We may take $\varepsilon=(s / 4) \lambda^{2 j_{0}}$ for instance. For $j \leq j_{0}$ we consider the sequence of functions defined iteratively by

$$
u_{j+1}(x, t)=\frac{1}{\lambda^{2}}\left(u_{j}(x, t)-\left(1-\lambda^{2}\right)\right),
$$

starting from $u_{0}(x, t)=u(x, t)$, the solution of the FPME under consideration. By induction we assume that

$$
\left(u_{j}\right)^{+}(x, t) \leq 1+\frac{1}{\lambda^{2 j}} \psi_{\varepsilon, \lambda}(x), \quad(x, t) \in \mathbb{R}^{N} \times(-3,0) .
$$

So for $j \leq j_{0}$ we have $u_{j} \leq 1+\psi_{\lambda}$. We recall that $\lambda$ is fixed and small. 
(ii) Next, we have to check the equation satisfied by $u_{j}$, which turns out to be

$$
u_{t}=\nabla\left(D_{j}(u) \nabla \mathcal{L}(u)\right)
$$

with a diffusion coefficient $D_{j}$ defined inductively by the rule

$$
D_{j+1}(s)=D_{j}\left(\lambda^{2} s+\left(1-\lambda^{2}\right)\right)
$$

so that $D_{j}\left(u_{j}\right)=D_{j+1}\left(u_{j+1}\right)$. This is the type of diffusion coefficients for which we have proved the modified estimates of Section 7 . We have to observe that, with the notations of that section, we have $d_{2} u_{j+1}=\lambda^{2} u_{j+1}=u_{j}-\left(1-\lambda^{2}\right)$ so that the integrals in $\mathcal{Q}$ do not change their form, in particular the formulas of the type (6.8), which depend on the integral

$$
\int \nabla L(x-y)\left(u_{j+1}(y)-\varphi_{k}(y)\right)^{-} d y,
$$

are estimated by the same constants. Note that $\int_{\mathbb{R}^{N}} \nabla L(x-y) C d y=0$ by antisymmetry of $\nabla L$.

(iii) By construction, the measure $\left|\left\{u_{j} \leq \varphi_{0}\right\} \cap\left(B_{1} \times(-4,-2)\right)\right|$ is increasing; hence, it is greater than $\rho$ for every $j$. We can apply Lemma 9.1 inductively to $u_{j}$. As long as $\left|\left\{u_{k}>\varphi_{2}\right\} \cap\left(\mathbb{R}^{N} \times(-2,0)\right)\right| \geq \delta$ for $k=1, \ldots, j+1$, we have

$$
\left|\left\{\varphi_{0} \leq u_{j+1} \leq \varphi_{2}\right\} \cap\left(\mathbb{R}^{N} \times(-3,0)\right)\right| \geq \gamma .
$$

Therefore, inside $\mathbb{R}^{N} \times(-3,0)$ we have

$$
\left|\left\{u_{j+1}>\varphi_{2}\right\}\right| \leq\left|\left\{u_{j+1}>\varphi_{0}\right\}\right|-\gamma \leq\left|\left\{u_{j}>\varphi_{2}\right\}\right|-\gamma \leq\left|B_{3} \times(-3,0)\right|-j \gamma .
$$

This cannot be true up to $j_{0}$, hence there must exist $j \leq j_{0}$ such that

$$
\left|\left\{u_{j}>\varphi_{2}\right\} \cap\left(\mathbb{R}^{N} \times(-2,0)\right)\right|<\delta .
$$

We can then apply the first lemma, Lemma 5.1, to the next step, $u_{j+1}$. Indeed,

$$
u_{j+1} \leq 1+\psi_{\lambda} \leq 1+\psi_{1} \quad \text { on } \mathbb{R}^{N} \times(-3,0)
$$

and

$$
\begin{aligned}
\left|\left\{u_{j+1}>1 / 2\right\} \cap\left(B_{2} \times(-2,0)\right)\right| & \leq\left|\left\{u_{j+1}>\varphi_{0}\right\} \cap\left(B_{2} \times(-2,0)\right)\right| \\
& \leq\left|\left\{u_{j}>\varphi_{2}\right\} \cap\left(\mathbb{R}^{N} \times(-2,0)\right)\right|<\delta,
\end{aligned}
$$

and then Lemma 5.1 implies that

$$
u_{j+1} \leq 1-\mu \quad \text { on } B_{1} \times(-1,0) .
$$

This gives the result with $\mu_{1}=\lambda^{2 j_{0}} \mu$. 


\section{End of proof of regularity for $s<1 / 2$}

The whole technical machinery is in place, and we are ready to prove Hölder regularity by means of the iterative process outlined in Section 5 . We take any point $P_{0}=\left(x_{0}, t_{0}\right) \in$ $\mathbb{R}^{N} \times(0, \infty)$ and prove that $u$ is $C^{\alpha}$ around $P_{0}$ with an exponent that depends only on the parameters $N$ and $s$ of the equation, and a Hölder constant that depends also on the $L^{\infty}$ norm of $u$ and a lower bound of $t_{0}$.

We start with some reductions. There is no loss of generality in assuming that $u$ is bounded in the cylinder $Q$ since we know by Theorem 1.1 that $u$ is bounded in any strip of the form $\mathbb{R}^{N} \times(\tau, \infty)$ with $\tau>0$. Moreover, by scaling we may assume that $t_{0}>4$. It will then be convenient to make a space-time translation and put $P_{0}=(0,0)$ assuming that the domain of definition of $u$ contains the strip $S_{4}=\mathbb{R}^{N} \times[-4,0]$. By another scaling we may also assume that $0 \leq u(x, t) \leq 1$ in $S_{4}$.

Consider now a positive constant $K<1 / 4$ such that the growth of the wings is controlled as follows:

$$
\frac{1}{1-\mu_{1} / 2} \psi_{\lambda, \varepsilon}(K x) \leq \psi_{\lambda, \varepsilon}(x)
$$

The coefficient $K$ depends only on $\lambda, \mu_{1}$ and $\varepsilon>0$. The parameters are as in the last section. The iteration that we will perform offers two possibilities.

- Alternative 1. Regularity at a degenerate point. Suppose that we can apply Lemma 10.1 repeatedly because the lowering of the oscillation may be assumed to happen always from above. We then consider the sequence of functions defined in the strip $S_{4}=\mathbb{R}^{N} \times[-4,0]$ by

$$
u_{j+1}(x, t)=\frac{1}{1-\mu_{1} / 4} u_{j}\left(K x, K_{1} t\right), \quad K_{1}=\frac{K^{2-2 s}}{1-\mu_{1} / 4} .
$$

Note that this time all the $u_{j}$ 's are solutions of the same equation. According to the running assumption, and using (11.1), we can apply Lemma 10.1 at every step so that we have $u_{j}(x, t) \leq 1-\mu_{1}$ in the cylinder $Q_{1}=B_{1} \times(-1,0)$ for every $j \geq 1$. In view of the scaling (11.2), this implies Hölder regularity around the point $(0,0)$, where the solution necessarily takes the degenerate value $u=0$ in a continuous way.

- Alternative 2. Regularity at points of positivity. After some steps of the iteration the assumption on the measure of the set $\left\{u_{j}>1 / 2\right\}$ made in Lemma 10.1 fails. Then we are in the situation where the oscillation is reduced from below thanks to Lemma 5.2, which pulls the solution uniformly up from zero in a smaller cylinder. Then the equation is no longer degenerate, because after that step we have

$$
0<\mu^{\prime} \leq u_{j}(x, t) \leq 1
$$

in the cylinder $B_{1} \times(-1,0)$. Scaling the situation we will be in the conditions of the equation with diffusivity $D(u)$ mentioned above, to which we apply either the modification of Lemma 10.1 or the modification of Lemma 5.2. In fact, we can apply the modification of Lemma 10.1 both from above and from below since the degeneracy has disappeared. In this way, we obtain Hölder regularity at a point $P_{0}$ where $u\left(P_{0}\right)>0$. 


\section{2. $C^{\alpha}$ regularity for $s>1 / 2$}

Here we want to prove the regularity result of Theorem 1.2 for $1 / 2<s<1$. If we try to re-do the proof of the technical oscillation lemmas listed in Section 5, we find a convergence problem that is already apparent in Lemma 5.1. Indeed, the bulk of the proof contained in Section 6 works without modification, and a problem was found only in the last estimate of Subsection 6.5, regarding the integral $\mathcal{Q}_{3}$ in an outer region, where the decay of the factors that we have been examining does not guarantee uniform convergence of the integrals if $s \geq 1 / 2$.

\subsection{Analysis of the difficulty}

A possible solution is to make use of the known fact that $u(x, t)$ is an $L^{1}$ function in $x$ uniformly in $t$, in order to bound the integral of the $y$ terms in $\mathcal{Q}_{3}$, with integrand $\nabla L(x-y) u_{k}^{-}(y)$, since $L(x-y)$ is bounded for large $|y|$. Indeed, we can put together the different parts of $\mathcal{Q}$ in the outer domain, and after dropping the contribution of the term in $u(x)$ since it is zero by the antisymmetry property of $\nabla L$, we get

$$
\begin{aligned}
& \left|\iint u_{k}^{+}(x) \frac{\nabla \varphi(x)}{\varphi(x)} \nabla L(x-y) u(y) d x d y\right| \\
& \quad \leq C\|u(\cdot, t)\|_{1}\left(\int u_{k}^{+}(x) d x\right) \leq C^{k}\|u(\cdot, t)\|_{1}\left(\int\left(u_{k-1}^{+}(x)\right)^{2} d x\right) .
\end{aligned}
$$

This solves the problem in one application of Lemma 5.1, but then $\delta$ and $\mu$ would depend on $\|u(t)\|_{1}$. However, to obtain the $C^{\alpha}$ regularity result we have seen in Section 11 that we need to iterate this and the other oscillation lemmas, we want to rescale and repeat, and then a difficulty reappears, because we will keep expanding the $u$ and the $x$ and therefore expanding the integral at every step, so the constants will be ruined in the iteration. We need a way to control such behaviour.

It will be convenient to examine the whole part of $\mathcal{Q}\left(u_{k}^{+}, u\right)$ that contains the difficulty, i.e.,

$$
\iint u_{k}^{+}(x) \frac{\nabla \varphi_{k}(x)}{\varphi_{k}(x)} \nabla L(x, y) u(y) d x d y .
$$

As we dilate and repeat in the iteration scheme, the term $\nabla L(x-y) u(y)$ also starts to build up as $y$ tends to infinity. On the other hand, the integrability in $y$ at infinity is lost if $s \geq 1 / 2$, since in that case $\nabla L$ decays like

$$
|\nabla L| \sim|y|^{-(N-2 s+1)}
$$

and this is not good enough. However, the good news is that

$$
\nabla \cdot(\nabla L) \sim|y|^{-(N+2-2 s)}
$$

(valid for all second derivatives), which is integrable as $|y| \rightarrow \infty$. Noting that $\nabla L$ is integrable for $y \sim 0$ if $s>1 / 2$, we conclude that

$$
V(x, t):=(\nabla L(x, y, t)) *_{y} u(y, t)
$$


has bounded Hölder seminorm. Therefore, it would be enough to control it at just one point, for instance at $x=0$ (for an interval of times).

\subsection{The transport approach for the case $s>1 / 2$}

The technical way to make use of the last observation is to perform a change of coordinates $x^{\prime}=x-S(t)$ that introduces a transport term to counter the difficult term $\int \nabla L(y) \Psi(y) u(y) d y$. Note that similar ideas have been used in [10, 18$]$.

We proceed as follows: We define

$$
S(t)=\int_{0}^{t} \vec{v}(s) d s, \quad \vec{v}(t)=\int \nabla L(y) u(y, t) d y,
$$

and we observe that $|\vec{v}(t)|$ depends also on $u$, and that $|\vec{v}(t)| \leq C<\infty$ since $u(y, t)$ is in $L_{y}^{1}$ uniformly in $t$. Indeed, the value of $\vec{v}(t)=\vec{v}(t ; u)$ is only controlled by the integral of $u$ in space (i.e., the mass of $u(t)$ ). Next, we introduce the change of variables

$$
(x, t) \mapsto\left(x^{\prime}, t^{\prime}\right):=(x-S(t), t),
$$

and we write the equation for $u$ with respect to the new variables, $u(x, t)=\tilde{u}\left(x^{\prime}, t^{\prime}\right)$. The RHS does not change since we are performing a space translation for fixed time. However, the time derivative on the LHS transforms as follows:

$$
u_{t}(x, t)=u_{t^{\prime}}\left(x^{\prime}+S\left(t^{\prime}\right), t^{\prime}\right)+(\nabla u) S^{\prime}\left(t^{\prime}\right)=\tilde{u}_{t^{\prime}}+\vec{v} \cdot \nabla_{x^{\prime}} \tilde{u} .
$$

The new term is what we are aiming at. The equation takes the convection-diffusion form

$$
\tilde{u}_{t}+\vec{v} \cdot \nabla_{x^{\prime}} \tilde{u}=\nabla(\tilde{u} \nabla \mathcal{L} \tilde{u}) .
$$

In the following we will write $t$ for $t^{\prime}$ and $u$ instead of $\tilde{u}$ without fear of confusion. The space variable is still written $x^{\prime}$. Next, we pass the term $\vec{v} \cdot \nabla u$ to the RHS and multiply by $\log ((u / \varphi) \vee 1)$, as we did in Section 6, to obtain the energy formula. We observe that in this case the RHS contains an extra term of the form

$$
I=\iint \nabla \log ((u / \varphi) \vee 1) \vec{v}(t) u\left(x^{\prime}, t\right) d x^{\prime} d t
$$

This integral must be computed only in the region where $u>\varphi$, and in that case $(u / \varphi) \vee 1$ $=u / \varphi=1+u_{k}^{+} / \varphi_{k}$, so that

$$
I=\int d t \int_{u>\varphi_{k}} \nabla u_{k}^{+} \vec{v}(t) d x^{\prime}-\int d t \int_{u>\varphi_{k}} u_{k}^{+} \frac{\nabla \varphi}{\varphi} \vec{v}(t) d x^{\prime}=I_{1}-I_{2} .
$$

The first integral vanishes, and the second is precisely the troublesome term:

$$
I_{2}=\int d t \int_{u>\varphi_{k}} d x\left(u_{k}^{+}\left(x^{\prime}\right) / \varphi_{k}\left(x^{\prime}\right)\right) \nabla \varphi_{k}\left(x^{\prime}\right) \int \nabla L\left(y^{\prime}\right) u\left(y^{\prime}, t\right) d y^{\prime},
$$


which disappears in this way from the calculation. Alternatively, the disappearance of the bad term in the energy calculation in the new variables can be easily seen if we write the equation for $\tilde{u}\left(x^{\prime}, t\right)$ in the more symmetrical form

$$
\tilde{u}_{t}=\nabla_{x^{\prime}}\left(\tilde{u}\left(\int\left\{\nabla L\left(y-x^{\prime}\right)-\nabla L(y)\right\}\left(\tilde{u}(y)-\tilde{u}\left(x^{\prime}\right)\right) d y\right)\right),
$$

to be interpreted in the same weak form, or weak energy form, that we used for $u(x, t)$. In any case, this allows us to prove Lemma 5.1 also for $s \in(1 / 2,1)$ if we work in the new coordinates, and the constants involved in the result do not depend on the $L^{1}$ norm of the solution. The price to pay is that the slope of the distorted space variables does depend on the $u$-integral. So, in the first step of the iteration process we have shown how to transfer the difficulty from a numerical term to a geometrical distortion.

In order to sum up the result, let us introduce the bound $M=1 \vee \sup _{t>0}|\vec{v}(t)|$, which depends only on $u$ via the norm $\sup _{t}\|u(\cdot, t)\|_{1}$.

Lemma 12.1. Let $1 / 2<s<1$ and let $u$ be a solution of the FPME under the assumptions of Lemma 5.1. Let us perform the above change of variables so that $\tilde{u}\left(x^{\prime}, t^{\prime}\right)$ is defined in the smaller cylinder $Q_{L}$ where $L=4 /(M+1)$. Then the result of Lemma 5.1 is true for $\tilde{u}$, with conclusion holding in a smaller cylinder $Q_{1 / M} ; \delta$ may depend also on $M$.

Thanks to (12.4), it is then immediate to see that the modified Lemma 7.1, as well as the pull-up Lemma 5.3 are also true if stated in the form that we have used for Lemma 12.1. A bit more attention to detail will show that the stronger reduction Lemma 10.1 also holds, since the iterations do not change the scaling in space and time.

\subsection{Analysis of the transport term in the final iteration}

When we try to perform again the iteration procedure of Section 11, one of the alternatives is repeated scaling around a degenerate point. In that case the iterations take the form

$$
u_{j+1}(x, t)=\frac{1}{1-\lambda_{*} / 4} u_{j}\left(K x, K_{1} t\right), \quad K_{1}=\frac{K^{2-2 s}}{1-\lambda_{*} / 4},
$$

which we may sum up as

$$
u_{1}\left(x_{1}, t_{1}\right)=A u(x, t), \quad x_{1}=B x, \quad t_{1}=C t,
$$

where $A<1, B<1$ and $C=B^{2-2 s} A$, so that the same equation will be satisfied after the change of scale. We propose here to do the same iteration for the solution $\tilde{u}$ in terms of the variables $x^{\prime}$ and $t$. The equation will then take the modified form (12.4), which will be satisfied again by the iterates, just as it is written. The velocity $\vec{v}(t)$ will change from iteration to iteration according to the rule

$$
\vec{v}_{1}(t)=\frac{C}{B} \vec{v}(C t)=A B^{1-2 s} \vec{v}(C t),
$$


which follows both from the geometric transformation, and from the definition of $\vec{v}$ in (12.2). Therefore, after the first geometrical transformation such repeated iterations conserve the same correspondence for all subsequent steps. In other words, the geometrical transformation done in the first step will hold for all remaining steps: if the set of coordinates at that moment is $\left(x_{n}, t_{n}\right)$, we obtain a set of newly distorted coordinates $\left(x_{n}^{\prime}, t_{n}\right)$ by the formula

$$
x_{n}^{\prime}(t)=x_{n}(t)-S_{n}(t), \quad S_{n}^{\prime}(t)=\vec{v}_{n}(t),
$$

which is just a scaled version of the original transformation for $n=0$. Summing up, since the contractions in the upper bound for $u$ happen with a constant rate $1-\mu$ in cylinders that shrink in space and time also with a fixed rate, we deduce in a standard way $C^{\alpha}$ regularity with respect to the transformed variables. But since the coordinate transformation is done only once and is Lipschitz continuous, this means the same type of Hölder regularity for $u$ with respect to the original coordinates $(x, t)$. Of course, the Lipschitz constant of the transformation depends on $M_{1}=\sup _{t}\|u(t)\|_{L_{x}^{1}}$.

The analysis of the second alternative is easier since we are converging along the iterations to an equation with constant diffusivity coefficient. We leave the easy details to the reader. This ends the proof of Theorem 1.2 for $1 / 2<s<1$.

\section{Extension of the existence theory}

After these results, we can extend the existence theory to all nonnegative and integrable initial data.

Theorem 13.1. For every $u_{0} \in L^{1}\left(\mathbb{R}^{N}\right), u_{0} \geq 0$, there exists a continuous weak solution of the FPME in the following sense: there exists a function $u(x, t)$, continuous and nonnegative in $Q=\mathbb{R}^{N} \times(0, \infty)$, such that

$$
\begin{gathered}
u \in L^{\infty}\left(0, \infty ; L^{1}\left(\mathbb{R}^{N}\right)\right) \cap L^{\infty}\left(\mathbb{R}^{N} \times(\tau, \infty)\right) \quad \text { for all } \tau>0, \\
\mathcal{L}(u) \in L^{1}\left(0, \infty ; W_{\mathrm{loc}}^{1,1}\left(\mathbb{R}^{N}\right)\right), \quad u \nabla \mathcal{L}(u) \in L^{1}(Q),
\end{gathered}
$$

and the identity

$$
\iint u\left(\eta_{t}-\nabla \mathcal{L}(u) \cdot \nabla \eta\right) d x d t+\int u_{0}(x) \eta(x, 0) d x=0
$$

holds for all continuously differentiable test functions $\eta$ in $Q$ that are compactly supported in the space variable and vanish near $t=\infty$.

Proof. (i) We take a sequence of initial data $u_{0 n}$ that are nonnegative, smooth and decaying at infinity as required in [11]. We assume that $\left\|u_{0 n}\right\|_{1} \leq C_{1}$ for all $n \geq 1$ and $u_{0 n} \rightarrow u_{0}$ in $L^{1}\left(\mathbb{R}^{N}\right)$. Then there exist solutions $u_{n}(x, t)$ and there are estimates like $u_{n} \geq 0$ and

$$
\int u_{n}(x, t) d x=\left\|u_{0 n}\right\|_{1} \leq C_{1} \quad \forall n \geq 1, t>0
$$


(ii) We also know from Theorem 1.1 that the solutions are bounded for $t \geq \tau>0$ with bound that depends only on the $L^{1}$ norm of the initial data:

$$
0 \leq u_{n}(x, t) \leq C(n, s)\left\|u_{0 n}\right\|_{1}^{\gamma} t^{-\alpha} \leq C C_{1}^{\gamma} t^{-\alpha} .
$$

We also know that uniformly bounded nonnegative solutions are $C^{\beta}$ smooth for some $\beta>0$ with uniform Hölder constants, so that after passing to a subsequence we have

$$
u_{n}(x, t) \rightarrow u(x, t) \quad \text { uniformly on compact subsets of } Q .
$$

(iii) We now observe that for every $t>0$ we have $u_{n}(t) \in L^{1} \cap L^{\infty}$, which together with the Sobolev embedding (or Riesz embedding) gives

$$
\mathcal{H}\left(u_{n}(t)\right) \in L^{r}\left(\mathbb{R}^{N}\right) \quad \forall r \geq N /(N-s)
$$

and the embedding is compact into $L_{\text {loc }}^{r}\left(\mathbb{R}^{N}\right)$. Indeed, if $u_{n} \in L^{p}$ then

$$
\frac{1}{r}=\frac{1}{p}-\frac{s}{N}, \quad p \geq 1 \text {. }
$$

In practice, we will need the estimate

$$
\int\left[\mathcal{H}\left(u_{n}(t)\right)\right]^{2} d x \leq C\left\|u_{n}(t)\right\|_{p}^{2}, \quad \frac{1}{2}=\frac{1}{p}-\frac{s}{N} .
$$

But since

$$
\left\|u_{n}(t)\right\|_{p}^{p} \leq\left\|u_{n}(t)\right\|_{1}\left\|u_{n}(t)\right\|_{\infty}^{p-1} \leq C\|u(t)\|_{1}^{1+(p-1) \gamma} t^{-\alpha(p-1)},
$$

we get the decay estimate for this energy in the form

$$
\int\left[\mathcal{H}\left(u_{n}(t)\right)\right]^{2} d x \leq C\|u(t)\|_{1}^{2(1+(p-1) \gamma) / p} t^{-2 \alpha(p-1) / p}=C\|u(t)\|_{1}^{\sigma} t^{\lambda-1},
$$

with

$$
\lambda=1-\frac{2 \alpha(p-1)}{p}=1-\frac{2 N}{N+2-2 s} \frac{N-2 s}{2 N}=1-\frac{N-2 s}{N+2-2 s}=\frac{2}{N+2-2 s} .
$$

(iv) We also have for every $t_{2} \geq t_{1} \geq 0$ the energy inequality

$$
\frac{1}{2} \int\left[\mathcal{H}\left(u_{n}\left(t_{2}\right)\right)\right]^{2} d x+\int_{t_{1}}^{t_{2}} \int u_{n}\left|\nabla \mathcal{L} u_{n}\right|^{2} d x d t \leq \frac{1}{2} \int\left[\mathcal{H}\left(u_{n}\left(t_{1}\right)\right)\right]^{2} d x .
$$

Therefore, we have a uniform estimate for the integral $\iint u_{n}\left|\nabla \mathcal{L} u_{n}\right|^{2} d x d t$ from $t=\tau$ $>0$ up to $t=\infty$, and we get the same decay rate.

(v) Next, we address the step of passing to the limit of the weak formulation of the solutions $u_{n}$ when the test function $\eta \in C^{1}(Q)$ is compactly supported in $Q$ away from $t=0$ :

$$
\iint u_{n}\left(\eta_{t}-\nabla \mathcal{L}\left(u_{n}\right) \cdot \nabla \eta\right) d x d t+\int u_{0 n}(x) \eta(x, 0) d x=0 .
$$


There is no problem in the convergence of $\iint u_{n} \eta_{t} d x d t$. If $\eta$ is supported away from $t=0$ then the second term converges since we can use the compactness of the map $u \mapsto$ $\nabla \mathcal{L}(u)$ from $L^{1} \cap L^{\infty}$ into $L^{r}$ for some $r$, due to the energy estimates plus embedding.

(vi) We now want to check that the initial data are taken. For that we show first that $u \nabla \mathcal{L} u \in L_{x, t}^{1}$ also near $t=0$. Here is the calculation in a strip $Q_{k}=\mathbb{R}^{N} \times\left(t_{k}, t_{k-1}\right)$ with $t_{k}=2^{-k}$ :

$$
\begin{aligned}
\iint_{Q_{k}} u|\nabla \mathcal{L}(u)| d x d t \leq\left(\iint_{Q_{k}} u d x d t\right)^{1 / 2}\left(\iint_{Q_{k}} u|\nabla \mathcal{L}(u)|^{2} d x d t\right)^{1 / 2} \\
\leq\left(\left\|u_{0}\right\|_{1} t_{k-1}\right)^{1 / 2}\left(\frac{1}{2} \int\left[\mathcal{H}\left(u\left(t_{k}\right)\right)\right]^{2} d x\right)^{1 / 2} \leq C t_{k}^{1 / 2} t_{k}^{(\lambda-1) / 2}=C t_{k}^{\lambda / 2} .
\end{aligned}
$$

With this it is possible to pass to the limit in the term $\iint u_{n} \nabla \mathcal{L}\left(u_{n}\right) \cdot \nabla \eta d x d t$ for $\eta$ that does not vanish near $t=0$ by estimating the integral for small $t$ as uniformly small, and then proving the convergence for $t \geq \tau>0$ using the known regularity. This proves that the definition of solution according to (13.1) applies to $u$.

(vii) We can also check that the initial data are taken as traces. This depends on a uniform estimate of the difference $u(\tau)-u_{0}$ for small $\tau$ in a suitable norm. For a $C_{0}^{1}$ test function $\eta(x)$ with support included in a bounded set $K$, we have

$$
\begin{aligned}
\int\left|u(\tau)-u_{0}\right| \eta d x & \leq \int_{0}^{\tau} \int\left|\partial_{t} u\right| \eta d x d t \leq \int u|\nabla \mathcal{L}(u)||\nabla \eta| d x d t \\
& \leq C\left(\iint_{K} u|\nabla \eta|^{2} d x d t\right)^{1 / 2}\left(\iint_{K} u|\nabla \mathcal{L}(u)|^{2} d x d t\right)^{1 / 2} \leq C \tau^{\lambda / 2}
\end{aligned}
$$

This is what makes $u(t) \rightarrow u_{0}$ in $W_{\text {loc }}^{-1,1}$ in the limit. Or we may use J. Simon's compactness results [19], since we are actually proving that $u_{t} \in L_{t}^{1}\left(W_{x}^{-1,1}\right)$.

Acknowledgments. Part of the present work was done during stays of F. Soria and J. L. Vázquez at Univ. of Texas in Austin, and of L. Caffarelli and J. L. Vázquez at MSRI, Berkeley, CA. L. Caffarelli has been funded by NSF Grant DMS-0654267 (Analytical and Geometrical Problems in Nonlinear Partial Differential Equations), F. Soria by Spanish Grant MTM2010-18128, and J. L. Vázquez by Spanish Grant MTM2011-24696.

\section{References}

[1] Aronson, D. G.: The porous medium equation. In: Nonlinear Diffusion Problems, Lecture Notes in Math. 1224, A. Fasano and M. Primicerio (eds.), Springer, New York, 1-46 (1986) Zbl 0626.76097 MR 0877986

[2] Bear, J.: Dynamics of Fluids in Porous Media. Dover, New York (1972) Zbl 1191.76001

[3] Biler, P., Karch, G., Monneau, R.: Nonlinear diffusion of dislocation density and self-similar solutions. Comm. Math. Phys. 294, 145-168 (2010) Zbl 1207.82049 MR 2575479 
[4] Boussinesq, J.: Recherches théoriques sur l'écoulement des nappes d'eau infiltrés dans le sol et sur le débit de sources. J. Math. Pures Appl. 10, 5-78 (1904) JFM 35.0761.01

[5] Caffarelli, L. A.: Further regularity for the Signorini problem. Comm. Partial Differential Equations 4, 1067-1075 (1979) Zbl 0427.35019 MR 0542512

[6] Caffarelli, L. A., Chan, Ch.-H., Vasseur, A.: Regularity theory for nonlinear integral operators. J. Amer. Math. Soc. 24, 849-869 (2011) Zbl 1223.35098 MR 2784330

[7] Caffarelli, L. A., Salsa, S., Silvestre, L.: Regularity estimates for the solution and the free boundary to the obstacle problem for the fractional Laplacian. Invent. Math. 171, 425-461 (2008) Zbl 1148.35097 MR 2367025

[8] Caffarelli, L. A., Silvestre, L.: An extension problem related to the fractional Laplacian. Comm. Partial Differential Equations 32, 1245-1260 (2007) Zbl 1143.26002 MR 2354493

[9] Caffarelli, L. A., Vasseur, A. F.: The De Giorgi method for regularity of solutions of elliptic equations and its applications to fluid dynamics. Discrete Contin. Dynam. Systems Ser. S 3, 409-427 (2010) Zbl 1210.76039 MR 2660718

[10] Caffarelli, L. A., Vasseur, A. F.: Drift diffusion equations with fractional diffusion and the quasi-geostrophic equation. Ann. of Math. 171, 1903-1930 (2010) Zbl 1204.35063 MR 2680400

[11] Caffarelli, L. A., Vázquez, J. L.: Nonlinear porous medium flow with fractional potential pressure. Arch. Ration. Mech. Anal. 202, 537-565 (2011) Zbl pre06101958 MR 2847534

[12] Caffarelli, L. A., Vázquez, J. L.: Asymptotic behaviour of a porous medium equation with fractional diffusion. Discrete Contin. Dynam. Syastems 29, 1393-1404 (2011) Zbl 1211.35043 MR 2773189

[13] Giacomin, G., Lebowitz, J. L.: Phase segregation dynamics in particle systems with long range interaction I. Macroscopic limits. J. Statist. Phys. 87, 37-61 (1997) Zbl 0937.82037 MR 1453735

[14] Giacomin, G., Lebowitz, J. L.: Phase segregation dynamics in particle systems with long range interaction II. Interface motion. SIAM J. Appl. Math. 58, 170729 (1998) Zbl 1015.82027 MR 1638739

[15] Giacomin, G., Lebowitz, J. L., Marra, R.: Macroscopic evolution of particle systems with short- and long-range interactions. Nonlinearity 13, 2143-2162 (2000) Zbl 0999.82051 MR 1794850

[16] Giacomin, G., Lebowitz, J. L., Presutti, E.: Deterministic and stochastic hydrodynamic equations arising from simple microscopic model systems. In: Stochastic Partial Differential Equations: Six Perspectives, Math. Surveys Monogr. 64, Amer. Math. Soc., Providence, RI, 107152 (1999) Zbl 0927.60060 MR 1661764

[17] Landkof, N. S.: Foundations of Modern Potential Theory. Grundlehren Math. Wiss. 180, Springer, New York (1972) Zbl 0253.31001 MR 0350027

[18] Silvestre, L.: Hölder estimates for advection fractional-diffusion equations. Ann. Scuola Norm. Sup. Pisa Cl. Sci. (5) 11, 843-855 (2012) Zbl pre06142474

[19] Simon, J.: Compact sets in the space $L^{p}(0, T ; B)$. Ann. Mat. Pura Appl. (4) 146 , 65-96 (1987) Zbl 0629.46031 MR 0916688

[20] Vázquez, J. L.: The Porous Medium Equation. Mathematical Theory. Oxford Math. Monogr., Clarendon Press, Oxford (2007) Z Zbl 1107.35003 MR 2286292

[21] Vázquez, J. L.: Smoothing and Decay Estimates for Nonlinear Diffusion Equations. Equations of Porous Medium Type. Oxford Lecture Ser. Math. Appl. 33, Oxford Univ. Press, Oxford (2006) Zbl 1113.35004 MR 2282669

[22] Vázquez, J. L.: Nonlinear diffusion with fractional laplacian operators.. In: Nonlinear Partial Differential Equations: the Abel Symposium 2010, H. Holden and K. H. Karlsen (eds.), Springer, 271-298 (2012) Zbl 1248.35223 